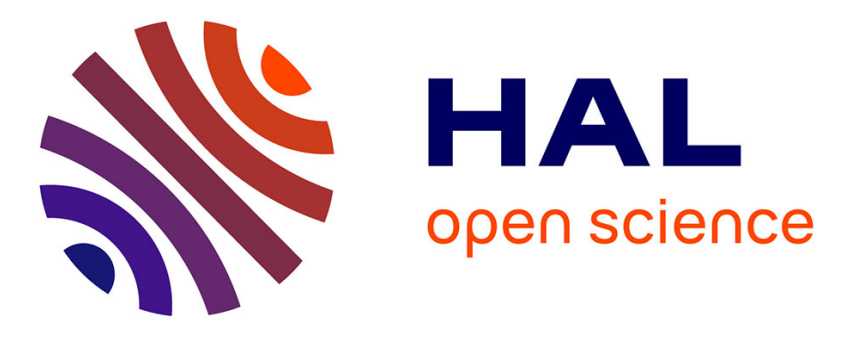

\title{
Insights into PPAR $\gamma$ phosphorylation and its inhibition mechanism
}

Roberta Montanari, Davide Capelli, Keiko Yamamoto, Hirono Awaishima, Kimina Nishikata, Arjan Barendregt, Albert J. R. Heck, Fulvio Loiodice, Fabio Altieri, Alessandro Paiardini, et al.

\section{To cite this version:}

Roberta Montanari, Davide Capelli, Keiko Yamamoto, Hirono Awaishima, Kimina Nishikata, et al.. Insights into PPAR $\gamma$ phosphorylation and its inhibition mechanism. Journal of Medicinal Chemistry, 2020, 63 (9), pp.4811-4823. 10.1021/acs.jmedchem.0c00048 . hal-03085021

\section{HAL Id: hal-03085021 https://hal.science/hal-03085021}

Submitted on 11 Jan 2021

HAL is a multi-disciplinary open access archive for the deposit and dissemination of scientific research documents, whether they are published or not. The documents may come from teaching and research institutions in France or abroad, or from public or private research centers.
L'archive ouverte pluridisciplinaire HAL, est destinée au dépôt et à la diffusion de documents scientifiques de niveau recherche, publiés ou non, émanant des établissements d'enseignement et de recherche français ou étrangers, des laboratoires publics ou privés. 


\section{Insights into PPAR $\gamma$ phosphorylation and its inhibition mechanism}

Roberta Montanari ${ }^{1 *}$, Davide Capelli ${ }^{1}$, Keiko Yamamoto $^{2}$, Hirono Awaishima $^{2}$, Kimina Nishikata ${ }^{2}$, Arjan Barendregt ${ }^{3}$, Albert J. R. Heck ${ }^{3}$, Fulvio Loiodice ${ }^{4}$, Fabio Altieri ${ }^{5}$, Alessandro Paiardini ${ }^{5}$, Alessandro Grottesi $^{6}$, Luciano Pirone ${ }^{7}$, Emilia Pedone ${ }^{7}$, Franck Peiretti ${ }^{8}$, Jean Michel Brunel $^{9}$, Toshimasa Itoh ${ }^{2} * \&$ Giorgio Pochetti ${ }^{1 *}$

${ }^{1}$ Istituto di Cristallografia, Consiglio Nazionale delle Ricerche, Via Salaria km. 29.300, 00015, Monterotondo Stazione, Rome, Italy

${ }^{2}$ Laboratory of Drug Design and Medicinal Chemistry, Showa Pharmaceutical University, 3-3165 Higashi-tamagawagakuen, Machida, Tokyo 194-8543, Japan

${ }^{3}$ Biomolecular Mass Spectrometry and Proteomics, Bijvoet Center for Biomolecular Research and Utrecht Institute for Pharmaceutical Sciences, Science4Life, University of Utrecht, Padualaan 8, 3584 CH Utrecht, The Netherlands

${ }^{4}$ Department of Pharmacy \& Drug Sciences, University of Bari "Aldo Moro", via Orabona 4, 70125, Bari, Italy

${ }^{5}$ Department of Biochemical Sciences “A. Rossi Fanelli”, Sapienza University of Rome, Piazzale Aldo Moro 5, 00185, Rome, Italy

${ }^{6}$ CINECA Consorzio Interuniversitario, Sede di Roma, 00185, Rome, Italy ${ }^{7}$ Institute of Biostructures and Bioimaging, CNR, Via Mezzocannone 16, 80134, Naples , Italy. 
${ }^{8}$ Aix Marseille Univ, INSERM, INRA, C2VN, Faculté de médecine, 13385, Marseille, France.

${ }^{9}$ Aix Marseille Univ, INSERM, SSA, MCT, 13385, Marseille, France

*Correspondence: giorgio.pochetti@ic.cnr.it (G.P.), titoh@ac.shoyaku.ac.jp (T.I.) and roberta.montanari@ic.cnr.it (R.M.)

\begin{abstract}
PPAR $\gamma$ represents a key target for the treatment of type II diabetes and metabolic syndrome. Synthetic antidiabetic drugs activating PPAR $\gamma$ are accompanied by serious undesirable side effects related to their agonism. In the search for new PPAR $\gamma$ regulators, inhibitors of PPAR $\gamma$ phosphorylation on S245 mediated by CDK5 represent an opportunity for the development of an improved generation of anti-diabetic drugs acting through this nuclear receptor. We have employed a multi-disciplinary approach, including protein-protein docking, X-ray crystallography, NMR, HDX, MD simulations and site-directed mutagenesis to investigate conformational changes in PPAR $\gamma$ that impair the ability of CDK5 to interact with PPAR $\gamma$ and hence inhibit PPAR $\gamma$ phosphorylation. Finally, we describe an alternate inhibition mechanism adopted by a ligand bound far from the phosphorylation site.
\end{abstract}




\section{Introduction}

The Peroxisome Proliferator-activated Receptors (PPARs) are transcription factors that regulate glucose and lipid metabolism. The role of PPARs in several chronic diseases such as type 2 diabetes (T2D), obesity and atherosclerosis, is well known ${ }^{1-3}$. Among the three subtypes $(\alpha, \beta / \delta$ and $\gamma)$ PPAR $\gamma$ is the most widely studied as therapeutically attractive target because of its key role in the regulation of energy balance and fat cell differentiation in the adipose tissue $e^{1,4-8}$. However, the initial clinical success of PPAR $\gamma$ agonists was soon overshadowed by several reports showing serious side effects $^{9,10}$, linked to their agonism, which turned the enthusiasm into scepticism. In 2010, Choi and co-workers' ${ }^{11,12}$ described a new class of antidiabetic compounds devoid of side effects. The authors observed that phosphorylation of PPAR $\gamma$ by the cyclin-dependent kinase 5 (CDK5) on S245 (S273 in PPAR $\gamma 2$ ) is linked to obesity and promotes loss of insulin sensitivity, although the same authors a few years later identified other kinases phosphorylating this site, such as $\mathrm{ERK}^{13}$. They described several new compounds that block CDK5-mediated phosphorylation avoiding the typical side effects caused by PPAR $\gamma$ full activation. However, the exact structural mechanism through which CDK5, in complex with its co-activator $\mathrm{p} 25$, is able to gain access to the PPAR $\gamma$ phosphorylation site, and what happens at structural level during PPAR $\gamma$ phosphorylation, have not yet been elucidated.

PPAR $\gamma$ is a 'functionally pluripotent' protein because its activity is mediated by ligands that differentially affect the structural conformation and the dynamic properties of the protein. As a consequence, different biological 
pathways can be activated depending on the coactivator/corepressor recruited and the differential propensity for CDK5 to phosphorylate S245. Thus, to design novel potent anti-diabetic agents, structural changes occurring during PPAR $\gamma$ phosphorylation need to be understood in detail. In this work we describe the mechanism underlying the CDK5/p25-mediated PPAR $\gamma$ phosphorylation providing a rationale for the inhibitory effect of certain ligands. In particular, we demonstrate that S245 within the putative PPAR $\gamma$ consensus motif SPFV (Supporting Information Figure 1) can only be accommodated in the active site of CDK5 through a transitory unfolding of the surface $\beta$-strand ( $\beta 1)$, close to the consensus region. Moreover, we show an alternate, allosteric mechanism to block PPAR $\gamma$ phosphorylation via a hydrophobic cross-talk pathway in which the amino-terminal portion of $\mathrm{H} 3$ plays a pivotal role. Ligands bound in the hydrophobic region between $\mathrm{H} 3$ and the $\beta$-sheet, such as the $R$ enantiomer of LT175 ${ }^{14}($ named $(R)-\mathbf{1}), \mathbf{7 j}^{15}$, $\mathbf{3 a}^{15}$, and MRL24 ${ }^{12,17}$, block phosphorylation through a direct stabilization of the hydrophobic region between $\mathrm{H} 3$ and $\beta 1-\beta 4$. In contrast, ligands such as the $S$ enantiomer of $\mathrm{LT} 175^{14,16}$, which bind far from the consensus motif, between $\mathrm{H} 11$ and H3, are also able to inhibit S245 phosphorylation through a long distance allosteric mechanism that results in stabilization of the $\beta$ sheet.

\section{Results}


To investigate the mechanism through which the CDK5/p25/ATP complex phosphorylates PPAR $\gamma$, we employed a range of in silico and solution-based approaches suitable for studying such a transient, but critical, interaction.

\section{Conformational dynamics within PPAR $\gamma$ are essential to enable phosphorylation of S245 by CDK5/p25}

In the past years, Mottin et al. ${ }^{18}$, by means of MD simulations, proposed four structural models of the complex between CDK5/p25 and PPAR $\gamma$-LBD. According to these models, the authors identified two distal regions (PPAR $\gamma$ $\Omega$-loop and $\beta$-sheet) in addition to the active site, that may play an important role in the interaction of CDK5 with the nuclear receptor. Among these models, the authors chose the most stable (model 3) as best candidate to represent PPAR $\gamma$-CDK5/p25/ATP interaction, where the RMSF of the residues of the phosphorylation site is very low during the entire simulation. Particularly, in the model proposed by Mottin et al., the external strand of the PPAR $\gamma \beta$-sheet is quite distorted, upon interaction with CDK5, with the disruption of one H-bond. Recently, Ribeiro Filho et al. ${ }^{19}$ proposed a quite different model of the complex between CDK5/p25 and PPAR $\gamma$-LBD. They found that PPAR $\gamma$ K261 occupies the $\mathrm{P}+3$ position of the consensus motif, forming a non-contiguous recognition site, as observed in other kinase substrates $^{20}$. In this position, K261 forms a salt-bridge with E240 residue of p25, and might be responsible for the Cdk5 specifity. Moreover, they identify other two lysines, K263 and K265, located at the flexible H2'-H3 loop of PPAR $\gamma$, pointing towards a region of Cdk5 that is rich in glutamic and aspartic residues (E/D rich region) and interacting with it through salt- 
bridges. They validated the role of these lysines as crucial anchor residues in the PPAR $\gamma / \mathrm{Cdk} 5$ interaction through single-point mutations (K261A, K263A and K265A), phosphorylation test and affinity studies. Finally, they identify by B-factor analysis a hydrophobic network of PPAR $\gamma$ residues (I341, M348, I249 and L255) which can be affected upon ligand binding, increasing the stability of $\mathrm{H} 2$ ' and $\mathrm{H} 2-\mathrm{H} 2$ ' loop and preventing a proper interaction with CDK5/p25. Particularly, they suggested a shift of the I341 side-chain, induced by the ligand, towards M348 ( $\beta$-sheet), I249 (H2-H2' loop) and L255 (H2') which could favor the stabilization of H2' helix, as observed in their B-factor analysis.

We also investigated the structural interaction between the CDK5/p25 complex and PPAR $\gamma$ by means of in silico protein-protein docking. The known crystal structures of both the complex between CDK2 and cyclin-A, with bound ATP and a short peptide substrate ${ }^{21}$ (PDB 1QMZ), and the complex between CDK5 and p25 without the substrate ${ }^{22}$ (PDB 1H4L), were selected as templates for building a model of CDK5 in complex with ATP and p25 (CDK2/CDK5 sequence identity: 60\%). Then, in order to model the interaction between CDK5/p25 and PPAR $\gamma$, the phosphorylation motif of PPAR $\gamma$ was superposed to the corresponding consensus motif of the known CDK2 peptide substrate. This initial rough complex was subsequently optimized using the Rosetta v.3.4 protein docking protocol ${ }^{23}$ (see Methods section for details).

The obtained predicted complex (Figure 1a) indicated that the accommodation of PPAR $\gamma$ Ser-Pro motif into the active site of CDK5 is feasible only upon enlargement of the loop comprising the targeted residue 
for phosphorylation, i.e. S245, and H-bond engagement of S245 with K128 of CDK5 (corresponding to the homologous active site residue K129 of CDK2). This implies, in turn, that the neighboring strand of the $\beta$-sheet $(\beta 1)$ must undergo a transient unfolding in order to bring S245 close to the CDK5 active site (Figure $1 b$ ). In this hypothetical process, three H-bonds would be transiently lost (F247CO-F347N, I249N-F347CO, I249CO-T349N), with a loss of free energy that could be compensated by the H-bonds engaged between PPAR $\gamma$ and CDK5, and by phosphorylation of S245.

The model proposed here has some structural similarities to model 3 of Mottin (Figure 1c), with the exception of the $\Omega$-loop conformation that is highly mobile. Particularly, model 3 of Mottin showed a partial unfolding of the surface $\beta 1$ strand of PPAR $\gamma$ that is in agreement with the results of our docking simulation, where this strand unfolds in a loop conformation. More interestingly, our model has striking similarities with that of Ribeiro Filho et al. ${ }^{19}$, in particular on the following points: a) the position $\mathrm{P}+3$ of the PPAR $\gamma$ consensus motif is also occupied by K261 (and not by V248) which forms a non-contiguous recognition site (S245-P246-F247-K261); b) K261, K263 and K265 are involved in electrostatic interactions with the Cdk5 E/D rich region by salt bridges; c) the $\Omega$-loop conformations of both models are very similar. Moreover, all the three proposed models evidenced the crucial position of $\mathrm{H} 2$ ' and $\mathrm{H} 2-\mathrm{H} 2$ ' loop at the interface between PPAR $\gamma$ and CDK5/p25 (Supporting Information Figure 2). 

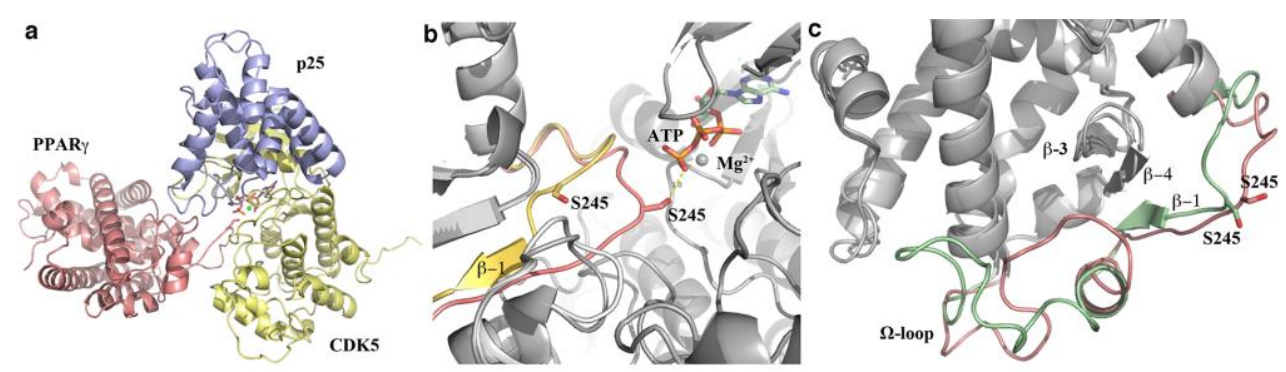

Figure 1. Docking study on the interaction between the CDK5/p25 complex and PPAR $\gamma$.

(a) Model of the complex PPAR $\gamma / \mathrm{CDK} 5 / \mathrm{p} 25 / \mathrm{ATP} / \mathrm{Mg}^{2+}$ (CDK5/p25: PDB code 1H4L; PPAR $\gamma$ : PDB code 1 PRG).

(b) Superposition of docked PPAR $\gamma$ (pink) and PPAR $\gamma$ (yellow) in its native state.

(c) Superposition of docked PPAR $\gamma$ (pink) to PPAR $\gamma$ (green) of the model 3 by M. Mottin (this model can be downloaded from the SI of ref.18).

\section{Direct link between stabilization of the $\beta$-sheet and S245 phosphorylation}

The double mutant F247C/G346C was expressed after a modelling study that confirmed the possibility to form a stable disulphide bridge between the two cysteines belonging to the $\beta$-sheet (Supporting Information Figure

3). The disulphide bridge was meant to strongly stabilize the $\beta$-sheet, avoiding the transient unfolding of the $\beta 1$ strand, with the aim to make S245 less available for the kinase. The mutant was equilibrated in the presence of a glutathione redox system $(0.3 \mathrm{mM}$ oxidized glutathione and $1.5 \mathrm{mM}$ reduced glutathione) to help the formation of the disulfide bridge ${ }^{24}$. The predominance of the oxidized form of the mutant was checked and confirmed by the Ellman's test that quantifies the presence of free sulfhydryl groups in solution (Supporting Information Table 1). The comparison between the CD spectra of F247C/G346C and PPAR $\gamma$ WT showed that both the proteins are correctly folded. In addition, the similarity of the CD spectra points to a very similar secondary structure content, with the preservation of 
the $\beta$-sheet (Supporting Information Figure 4 and Table 2). The further step was to perform the phosphorylation test checking the degree of phosphorylation of the double mutant. For detecting PPAR $\gamma$ phosphorylation rate the Pro-Q Diamond Phosphoprotein Gel Stain method has been used (see Experimental Section), avoiding in this way the use of specific antibodies. The results of the test (Figure 2) showed that the strong stabilization of the $\beta$-sheet prevents the phosphorylation of S245. This implies that different degrees of stabilization of the $\beta$-sheet by PPAR $\gamma$ ligands, full or partial agonists, may inhibit the S245 phosphorylation in a graded way. To check if the double mutation could affect itself the phosphorylation of S245, regardless the formation of the disulphide bridge, the mutant was equilibrated in a buffer containing TCEP $1 \mathrm{mM}$ in order to obtain a form of the protein with predominant free sulfhydryls. This form is also folded as the oxidized one (Supporting Information Figure 4 and Table 2). In Figure 2 it is shown that the predominant reduced form of the mutant (after equilibration with TCEP $1 \mathrm{mM}$ ) is able, unlike the oxidized form, to phosphorylate S245 as the WT, although to a lesser degree.

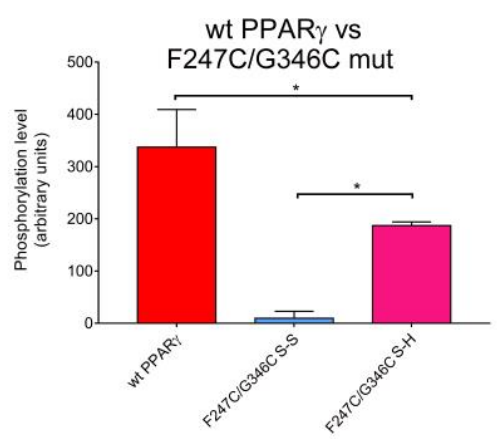


Figure 2. Phosphorylation level of double mutant F247C/G346C PPAR $\gamma$.

Comparison between S245 phosphorylation of WT PPAR $\gamma$, F247C/G346C (S-S) PPAR $\gamma$

and F247C/G346C (SH) PPAR $\gamma$. Phosphorylation level was evaluated by using the Phospho Gel Stain method. For each condition tested the intensity of the protein incubated in absence of kinase was subtracted to that obtained in presence of kinase and values obtained were normalized to the amount of protein loaded. Data represent the mean of at least three measurements and error bars indicate SD. Ligand concentrations were $10 \mu \mathrm{M}$. All data were analyzed by one-way ANOVA with multiple comparisons followed by Dunnet test. In all cases, $\mathrm{P}<0.05$ was considered statistically significant $(*=\mathrm{P} \leq 0.05, * * *=\mathrm{P} \leq 0.01)$.

\section{LT175 is an allosteric inhibitor of PPAR $\gamma$ phosphorylation}

The following step was to test the capability to inhibit the S245 phosphorylation of some PPAR $\gamma$ ligands (7j, 3a, MRL24, LT175 and rosiglitazone) whose different binding mode to the PPAR $\gamma$ LBD is known.
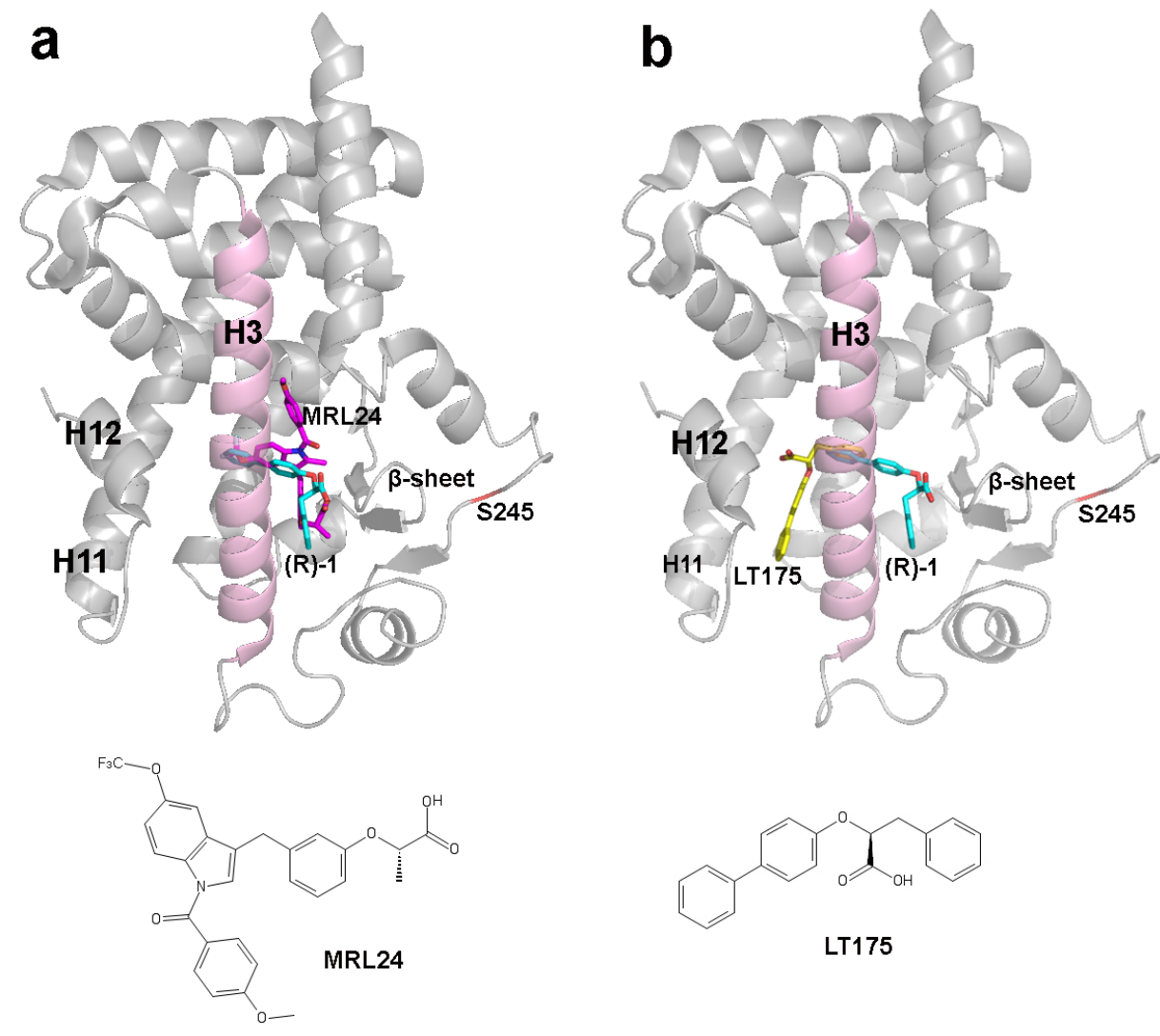

Figure 3. Comparison between different ligand binding modes in the PPAR $\gamma$-LBD. 
(a) superposition of $(R)$-1, (cyan) and MRL24 (purple). (b) Superposition of LT175 (yellow) and (R)1. The phosphorylation target, S245, is shown in red, helix 3 in pink and the $\beta$-sheet in light blue. (PDB codes of LT175, $(R)-\mathbf{1}$ and MRL24 structures are 3B3K, 3D6D and 2Q5P, respectively).

Starting from the observation that $\mathbf{7} \mathbf{j}$ and $\mathbf{3 a}$ have a very similar structure and bind similarly to PPAR $\gamma$, unless their terminal aliphatic chain that in $\mathbf{7 j}$ faces the $\beta$-sheet whereas in 3a occupies a different region of the LBD (Supporting Information Figure 5), we tested their potency as inhibitors of PPAR $\gamma$ phosphorylation with the aim to observe if the different stabilization of the $\beta$-sheet could affect the phosphorylation degree. The results showed that $\mathbf{7 j}$, able to better stabilize the $\beta$-sheet with both disordered conformations of its aliphatic chain, inhibits the phosphorylation to a greater extent than 3a (phosphorylation level of 14\% vs 36\%) (Figure 4 upper panel).

MRL24, that also occupy the same region of $\mathbf{7} \mathbf{j}$ aliphatic chain (Figure 3a) between $\mathrm{H} 3$ and the $\beta$-sheet, strongly inhibits the phosphorylation (32\%). Surprisingly, LT175, which binds PPAR $\gamma$ LBD in the region between H1 1 and $\mathrm{H} 3$, far from the phosphorylation site (Figure 3b), also inhibits the phosphorylation and better than the reference molecule rosiglitazone ${ }^{25}$ (phosphorylation level of $47 \%$ vs $65 \%$ ). These results were also confirmed by an ELISA protocol (enzyme-linked immunosorbent assay) where the inhibition by LT175 was comparable to that by its enantiomer $(R)-\mathbf{1}$, bound to the LBD similarly to MRL24 (Supporting Information Figure 6a). The result of these experiments let us hypothesize a long-distance inhibition mechanism of PPAR $\gamma$ phosphorylation induced by LT175. 

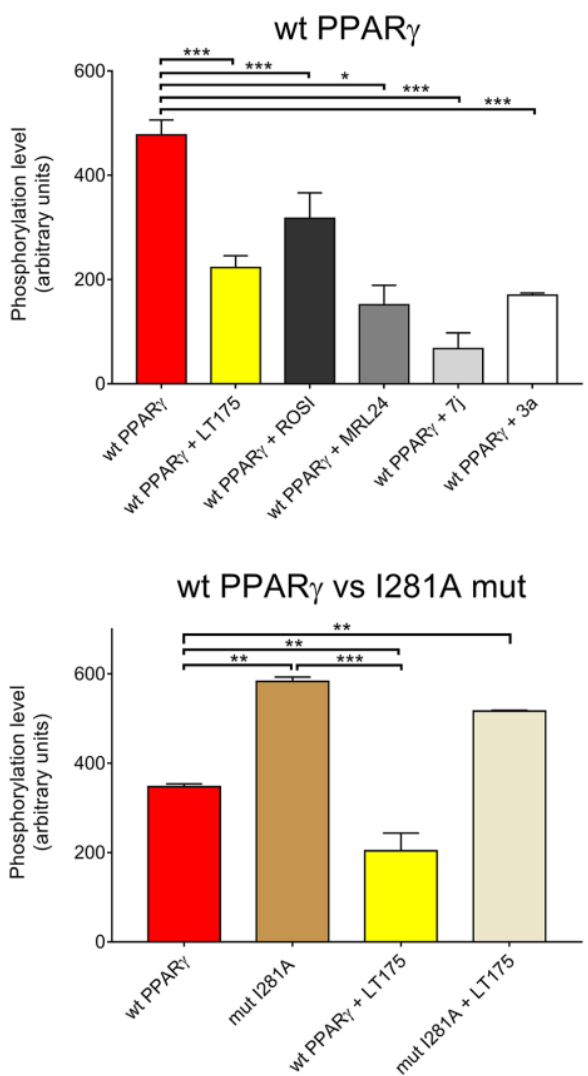

Figure 4. Phosphorylation level of PPAR $\gamma$ and I281A mutant in the presence of different ligands.

Upper panel: inhibition of the phosphorylation on wt PPAR $\gamma$ LBD by LT175 and other ligands. Lower panel: inhibition of the phosphorylation on the mutant I281A LBD by LT175. The phosphorylation degree of I281A was ca. 60\% higher than the WT, confirming that the mutation doesn't prevent the phosphorylation. Phosphorylation level was evaluated by using the Phospho Gel Stain method. For each condition tested the intensity of the protein incubated in absence of kinase was subtracted to that obtained in presence of kinase and values obtained were normalized to the amount of protein loaded. Data represent the mean of at least three measurements and error bars indicate SD. Ligand concentrations were $10 \mu \mathrm{M}$. All data were analyzed by one-way ANOVA with multiple comparisons followed by Dunnet test. In all cases, $\mathrm{P}<0.05$ was considered statistically significant $(*=\mathrm{P} \leq 0.05, * * *=\mathrm{P} \leq 0.01)$.

The Supporting Information Figure $6 \mathrm{~b}$ shows the gel with the experiment of phosphorylation on PPAR $\gamma$ WT and the mutant I281A with LT175, MRL24 and Rosi (Phospho Gel Stain method). The Supporting Information Figure 7 shows the phosphorylation of Histone H1, with or without LT175, and demonstrates that the ligand doesn't inhibit Cdk5 through a direct interaction 
Structural mapping of the allosteric inhibition mechanism of PPAR $\gamma$ phosphorylation by CDK5

We carried out a $2 \mathrm{D}{ }^{1} \mathrm{H}^{-15} \mathrm{~N}$ HSQC NMR experiment to map the effect of the ligand binding event on the PPAR $\gamma$ backbone chemical shifts and compared the spectra with that of apo-PPAR $\gamma$. For this aim we analysed several compounds with different potency and bound to different regions of the PPAR $\gamma$ LBD (LT175, GW192926, rosiglitazone) (Figure 5a). 

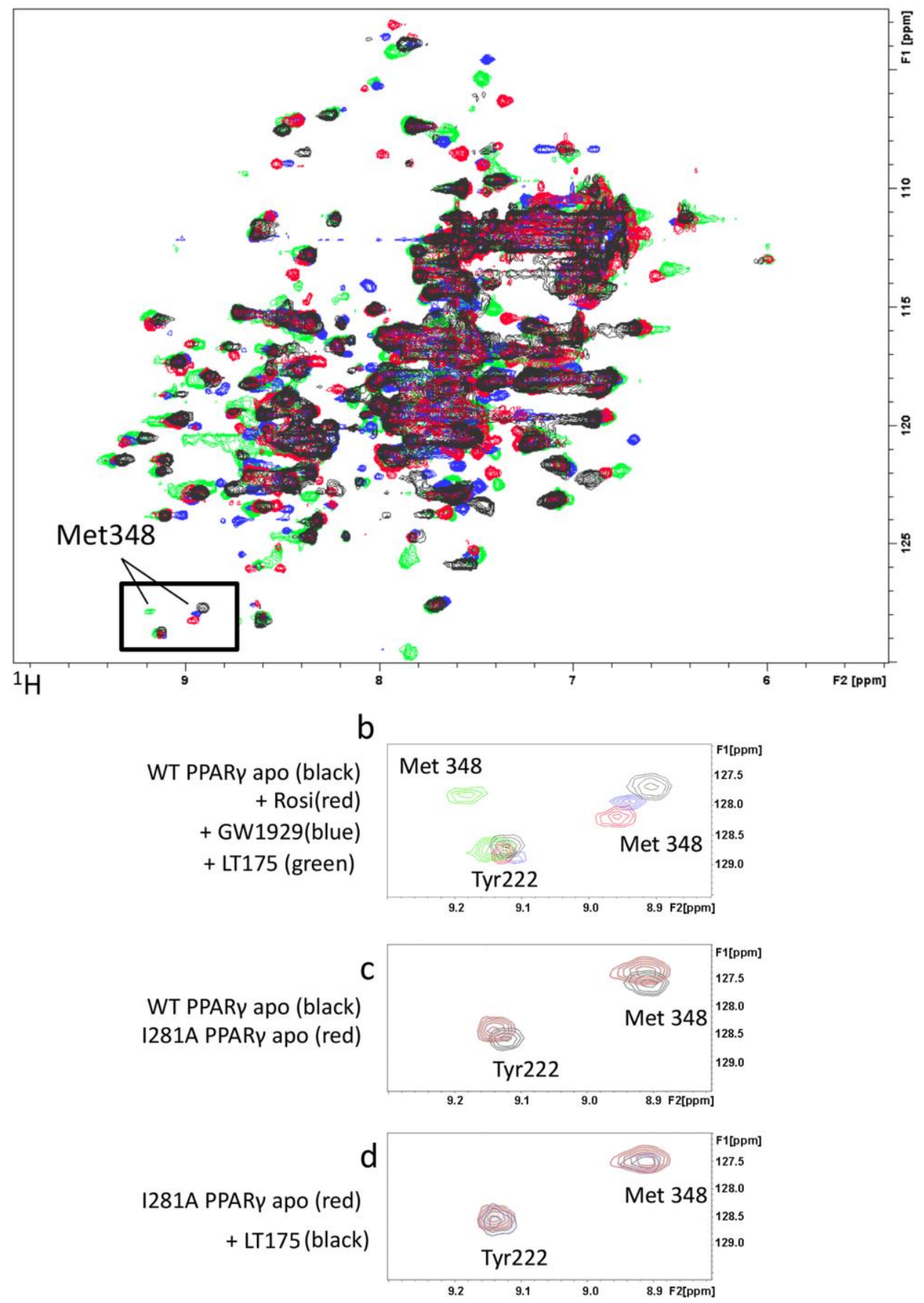

Figure 5. ${ }^{1} \mathrm{H}-{ }^{15} \mathrm{~N}$ HSQC NMR spectra.

(a) ${ }^{1} \mathrm{H}-{ }^{15} \mathrm{~N}$ HSQC NMR spectra: black, blue, red and green peaks show apo PPAR $\gamma$-LBD, PPAR $\gamma+\mathrm{GW} 1929$, PPAR $\gamma+$ rosiglitazone and PPAR $\gamma+\mathrm{LT} 175$, respectively; (b) superimposed ${ }^{1} \mathrm{H}-{ }^{15} \mathrm{~N}$ HSQC spectra of WT PPAR $\gamma$ apo (black), PPAR $\gamma+$ rosiglitazone (red), PPAR $\gamma+$ GW1929 (blue) and PPAR $\gamma+\mathrm{LT} 175$ (green); (c) superimposed ${ }^{1} \mathrm{H}-{ }^{15} \mathrm{~N}$ HSQC spectra of WT PPAR $\gamma$ apo (black) and I281A mutant apo (red); (d) superimposed ${ }^{1} \mathrm{H}^{-15} \mathrm{~N}$ HSQC spectra of I281A mutant apo (red) and in presence of LT175 (black). The concentration of the ligands was 1 molar equivalent. 
We focused our attention on the cross-peak related to M348, a residue located on the $\beta 4$ strand, directly linked to helix 3 through vdW interactions (about $4 \AA$ from I281). The M348 cross-peak, that could be easily assigned in the spectrum, was slightly shifted in the presence of rosiglitazone, as already observed by T.S. Hughes and co-workers ${ }^{27}$, and of GW1929 (Figure $5 b)$. This is in accordance with the observation that both ligands have an aromatic ring close to the M348 side-chain. In the presence of LT175, bound very far from the M348 side-chain and the phosphorylatable S245 (about

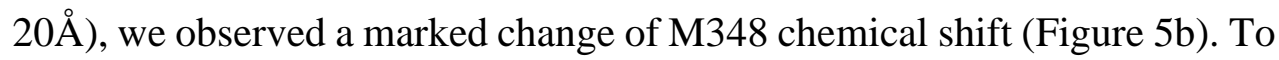
go more in depth, we performed titration experiments with LT175 ranging from 0.1 to 1 molar equivalent and we observed the M348 peak getting gradually weaker in intensity until disappearing at 1 molar equivalent, and the concomitant appearance of a new M348 peak in the spectrum (Supporting Information Figure 8). This suggested that M348, despite the long distance from the ligand, is strongly affected by LT175 binding through a hydrophobic cross-talk pathway running from the ligand up to the $\beta$-sheet, via $\mathrm{I} 281$ on $\mathrm{H} 3$.

The central position of I281 in this pathway led us to hypothesize a crucial role of this residue in the molecular cross-talk and that its mutation to I281 A might interrupt the hypothesized signaling mechanism. We then mutated I281 to Ala and compared the NMR spectrum of apo-I281A to that of apoWT, observing only a negligible change in the chemical shift of M348 and the close Y222 (Figure 5c).

When comparing the spectrum of I281A PPAR $\gamma$ apo to that of its complex with LT175 we observed that the M348 cross-peak did not shift (Figure 5d). 
This confirms that the residue I281 could be crucial for the signaling pathway. We performed SPR experiments to confirm that LT175 also binds to the I281A mutant with similar affinity to that of WT. The SPR results (Supporting Information Figure 9) show that LT175 binds I281A and WT with a similar affinity $\left(\mathrm{K}_{\mathrm{a}}\right.$ of $3.7 \mathrm{E} 5$ versus $\left.5.5 \mathrm{E} 5 \mathrm{M}^{-1}\right)$. The kinetic data (Figure 9 of Supporting Information) indicate a greater flexibility of the mutant as LT175 associates and dissociates faster with the mutant than with WT (3 and 4 times, respectively).

To investigate the stability of the holo mutant, Circular Dichroism (CD) experiments were then performed. CD shows that the mutant is as stable as the WT but in the presence of the ligand it is stabilized much weaker than WT, which, on the contrary, results strongly stabilized (Figure 6a,b). Also these data reflect an allosteric structural coupling via I281 between the ligand binding region, delimited by $\mathrm{H} 3$ and $\mathrm{H} 11$, and the $\beta$-sheet.

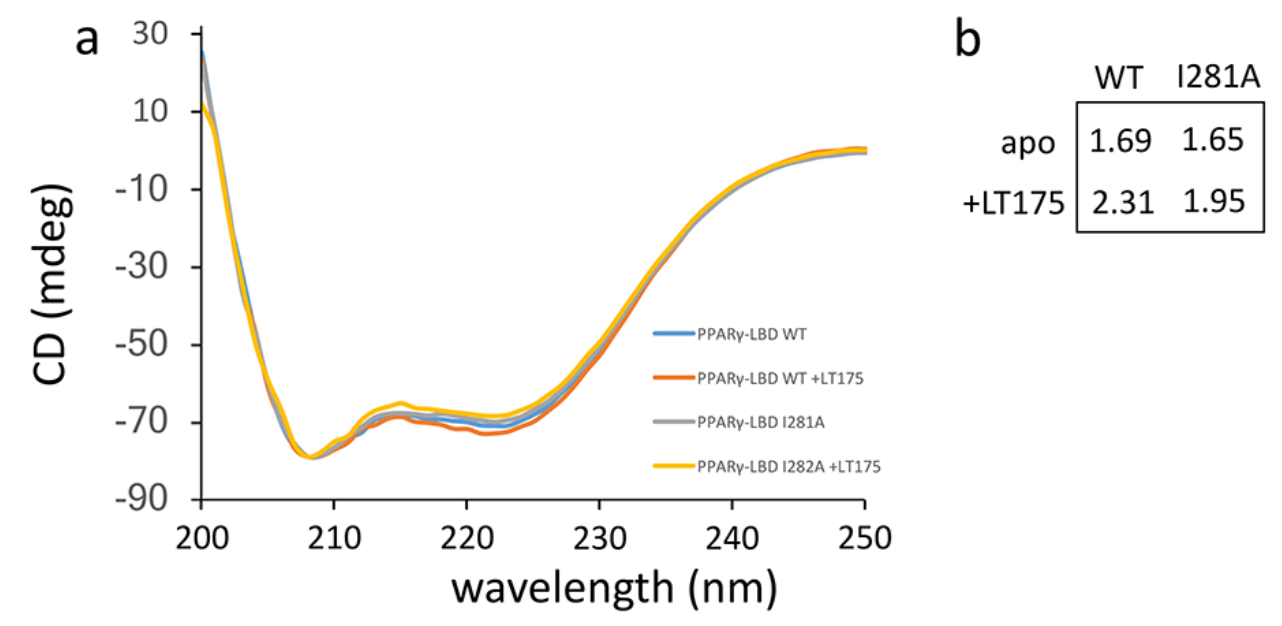

Figure 6. CD spectra.

Biophysical characterization and evaluation of PPAR $\gamma$ WT and I281A with or without LT175. (a) PPAR $\gamma(20 \mu \mathrm{M}) \quad$ spectra of Circular Dichroism (CD) from 200 to $250 \mathrm{~nm}$. (b) Results of denaturation experiment using $\mathrm{GdmCl}(0,0.5,1.0,2.0,3.0,6.0 \mathrm{M})$. Each value indicates guanidine concentration to achieve $50 \%$ of folded protein, calculated by Boltzmann function in the software Prism. 
On the basis of the so far acquired experimental and computational data, a cross-talk mechanism has been hypothesized as shown in Figure 7. As already known, upon binding of LT175, the side-chain of F282 is considerably shifted from its original position (Supporting Information Figure 10) as observed in the apo-form, allowing a vdW interaction with L356 side-chain ${ }^{14}$. This would trigger the signal, via L356, L353 and I281, up to M348 on the strand $\beta 4$, I249 on $\beta 1$, and L255 on H2' (Figure 7).

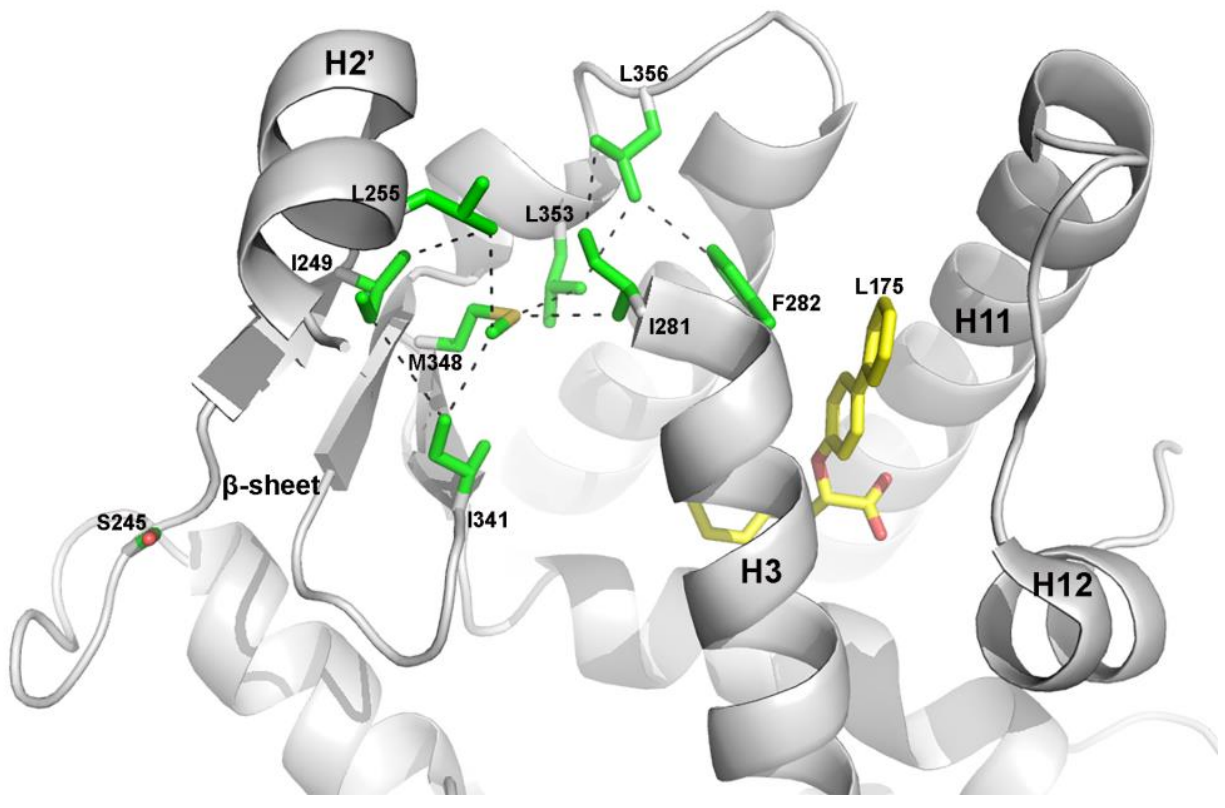

Figure 7. Hypothesis of allosteric inhibition by LT175.

Hypothesized hydrophobic signaling pathway from the ligand to S245.

\section{I281 has a pivotal role in allosteric blocking of PPAR $\gamma$ phosphorylation}

In order to confirm the proposed pathway from F282 to S245 we repeated the phosphorylation assay on the mutant I281A. 
Our attempts to mutate other residues involved in the cross-talk pathway were fruitless because the mutants were unstable or difficult to express. Conversely, the I281A mutant has the same stability as wild-type PPAR $\gamma$, as shown by $\mathrm{CD}$ results (Figure 6a,b).

Consistent with our hypothesis, we observed in the mutated protein a significant decreasing of the phosphorylation inhibition upon ligand binding (Figure 4 lower panel and Supporting Information Figure 6b), demonstrating that a break in the molecular cross-talk pathway disfavours the stabilization of the $\beta$-sheet and lowers the inhibitory potency of LT175. It is important to observe that I281A mantains the ability to phosphorylate S245, even to a greater extent than the WT as shown in the Figure 4 lower panel.

\section{LT175 stabilizes H3}

Molecular Dynamics simulations of the wild-type PPAR $\gamma$ in its apo- and holo-form with LT175 were carried out to study conformational dynamics of the PPAR $\gamma$ structure with and without ligand. To this end, a $200 \mathrm{~ns}$ simulation, typically, of both the apo and holo PPAR $\gamma$ were performed to investigate the role played by the key residues Phe-282 and Ile-281, belonging to H3, in PPAR $\gamma$ stability and to confirm the hypothesized hydrophobic pathway from the ligand to the $\beta$-sheet and S245, via H3. Analysis of the equilibrated part of the simulations (see Methods) showed that global flexibility (RMSF) of wild type PPAR $\gamma$ in the presence of ligand was reduced with respect to the apo counterpart. As shown in Figure 8a, a general decrease of the RMSF values was observed upon ligand binding for the wild-type PPAR $\gamma$ throughout its structure, the only 
exception being a short loop encompassing residues 269-274 ( $\omega$-loop), thus suggesting that the reduced accessibility of the phosphorylated Ser245 to the bulk solvent could be ascribed to the increased compactness of the protein structure.

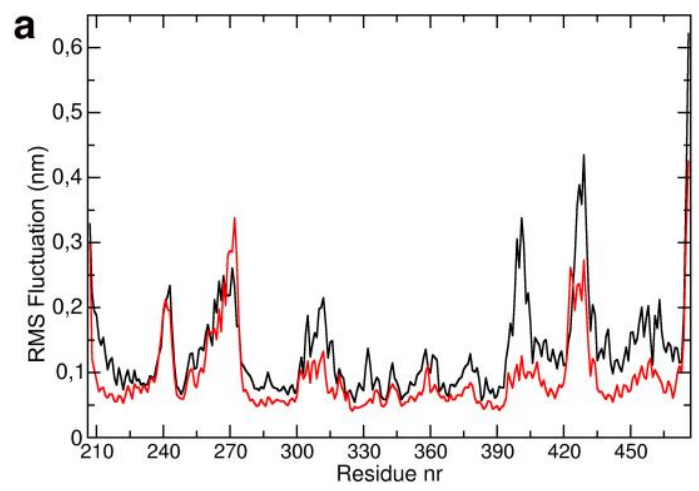

b

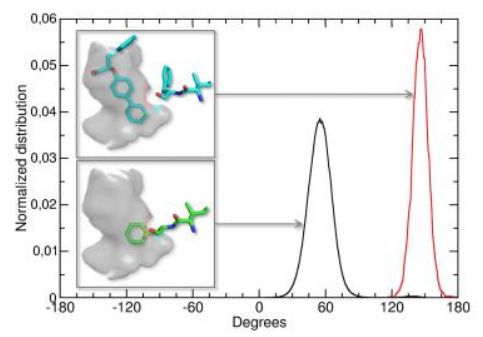

c

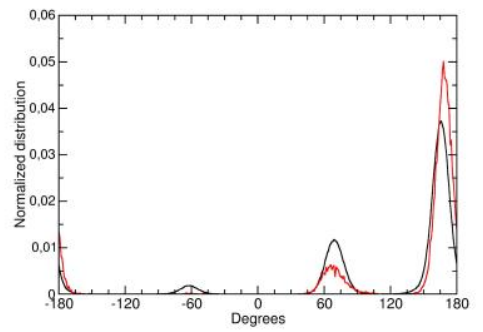

Figure 8. Molecular dynamics simulation.

(a) RMSF (nm) of alpha carbon atoms as function of residue number;

(b) Phe $282 \chi_{1}$ dihedral angles distribution, as calculated on the equilibrated part of all simulations. (c) I281 $\chi_{1}$ dihedral angles distribution.A schematic representation of Phe-282 average position for the two distribution is also shown in the insets. (black: apo PPAR $\gamma$, red: holo PPAR $\gamma$ with LT175).

To get a deeper insight into the molecular mechanism of protein stabilization upon ligand binding, the role played by specific residues connecting the ligand binding pocket to the phosphorylated loop hosting Ser-245 was investigated by dihedral angles analysis throughout the simulations. The results are shown in Figure $8 b, c$.

The figure reports the population distribution of the $\chi_{1}$ dihedral angles in the equilibrated portions of the trajectories for Phe-282 and Ile-281. As shown, Phe-282 adopts two distinct $\chi_{1}$ population distributions with and 
without ligand. This suggests that in apo-PPAR $\gamma$ the Phe-282 side-chain is located in correspondence of the phenyl moiety of LT175, while binding of LT175 forces Phe-282 side-chain to adopt a different conformation, where the phenyl ring makes strong hydrophobic contacts with Ile-281.

In turn, the distribution of $\chi_{1}$ populations of Ile-281 revealed a more complex behaviour: in apo-PPAR $\gamma$ Ile-281 $\chi_{1}$ adopts 3 different modal values (i.e. -60, 60, and 180); upon ligand binding and engaging hydrophobic interactions with Phe-282, the distribution of the Ile-281 $\chi_{1}$ angles is restrained to a bimodal value, being the population centered at 60 degrees almost canceled out. This suggests that LT175 binding to PPAR $\gamma$ causes a re-arrangement of the PPAR $\gamma$ hydrophobic interactions at the Ile-281/Phe-282 site, resulting in a reduction of the conformational freedom of Ile-281. The latter is indeed at contact distance with Met-348, which belongs to a cluster of hydrophobic residues (including M348, I341 and I249) connecting the $\beta$-sheet to the loop hosting Ser-245. The change in conformational dynamics of PPAR $\gamma$ upon LT175 binding was detected by analyzing the cross-correlations and concerted motions calculated using Essential Dynamics (ED) that provides principal directions of fluctuations where protein motion is likely to occur. ED analysis showed that the reduction in protein conformational flexibility by ligand binding was not only a global effect on PPAR $\gamma$ (Supporting Information Movie $1,2)$ but it affects specifically the structural integrity of the strand $\beta 1$, close to Ser-245, thus affecting its phosphorylation rate as well. Therefore, the MD results seem to confirm our hypothesis of the hydrophobic cross- 
talk pathway running from the ligand LT175 up to the S245, via I281 on H3 and M348 on $\beta 4$.

\section{HDX-MS}

Hydrogen/deuterium exchange/mass spectrometry ${ }^{28}$ (HDX-MS) was used to also probe the conformational changes remote from the ligand binding site with the aim to confirm the allosteric mechanism of inhibition of CDK5mediated PPAR $\gamma$ phosphorylation by LT175. We ran the experiment on both WT PPAR $\gamma$ and I281A PPAR $\gamma$ in order to probe the pivotal role of this residue in the hydrophobic signaling pathway.

Exchange dynamics of various segments was monitored by measuring the deuterium content of the corresponding pepsin induced peptide fragments. A sequence coverage of $92.4 \%$ was achieved (Supporting Information Figure 11). Comparative H/D exchange heat maps of WT PPAR $\gamma$ in the presence or absence of LT175 did overall not reveal any significant changes, with the exception of two specific regions (Figure 9a).

In the region 277-286, corresponding to the amino-terminal portion of $\mathrm{H} 3$ and containing the residue I281, the deuterium uptake decreases considerably in the presence of LT175, whereas these effects are not observed in mutated PPAR $\gamma$ I281A (Figure 9b). This observation confirms the key role of both the initial part of $\mathrm{H} 3$ and the residue I281 in the molecular cross-talk pathway. As known, the binding of LT175 in the 'diphenyl pocket' provokes the conformational switch of F282 side-chain from trans to gauche* conformation (Supporting Information Figure 10) and in turn a vdW interaction of this residue with the L356 side-chain (Figure 7). 
The mutation I281A breaks this interaction making this region less stable, as confirmed by our HDX. The other change in deuterium uptake concerns H12, that, as expected, is better stabilized by LT175 through H-bonds with Y473 (Figure 9a,b and Supporting Information Figure 12). Interestingly, LT175 is not able to stabilize H12 in the mutant I281A (Figure $9 \mathrm{~b}$ and Supporting Information Figure 12), demonstrating once more the importance of the initial part of $\mathrm{H} 3$ (I281 and F282) as fundamental pivot in the communication among distant regions of the LBD.

A minor but significant change in the deuterium uptake was observed in the region of the external $(\beta 1)$ strand of the $\beta$-sheet. Considering the relative fractional uptake of PPAR $\gamma$ and PPAR $\gamma+\mathrm{CDK} 5$ in the range $+15.0 \%$ to $+40.0 \%$, it is evident that LT175 stabilizes this region in PPAR $\gamma$ WT but not in the mutant (Figure 9c). This confirms that the mutation I281A breaks the molecular cross-talk pathway (Figure 7). 
a

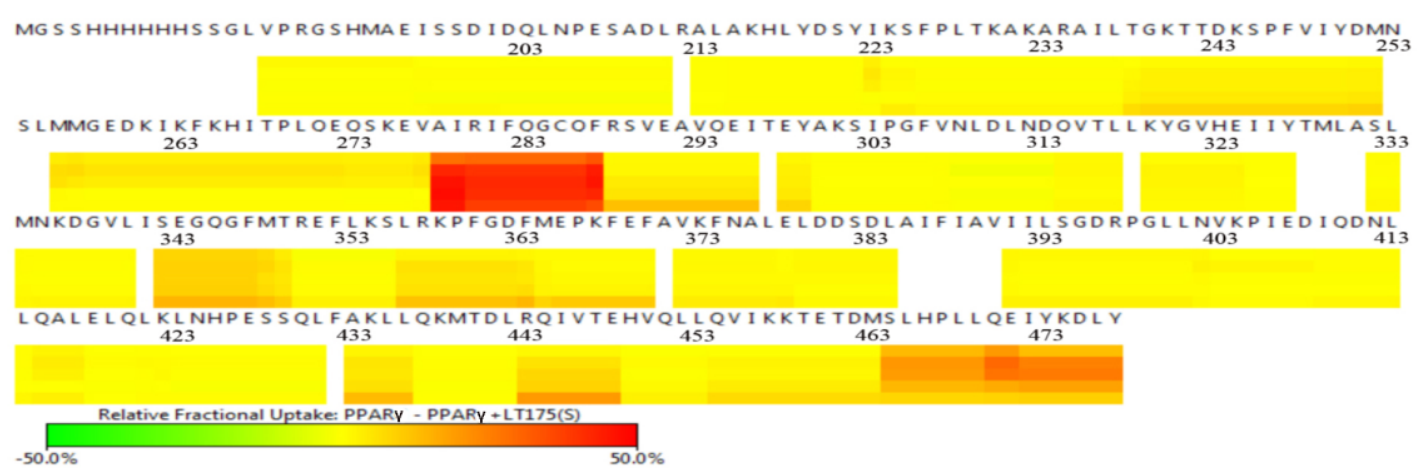

b
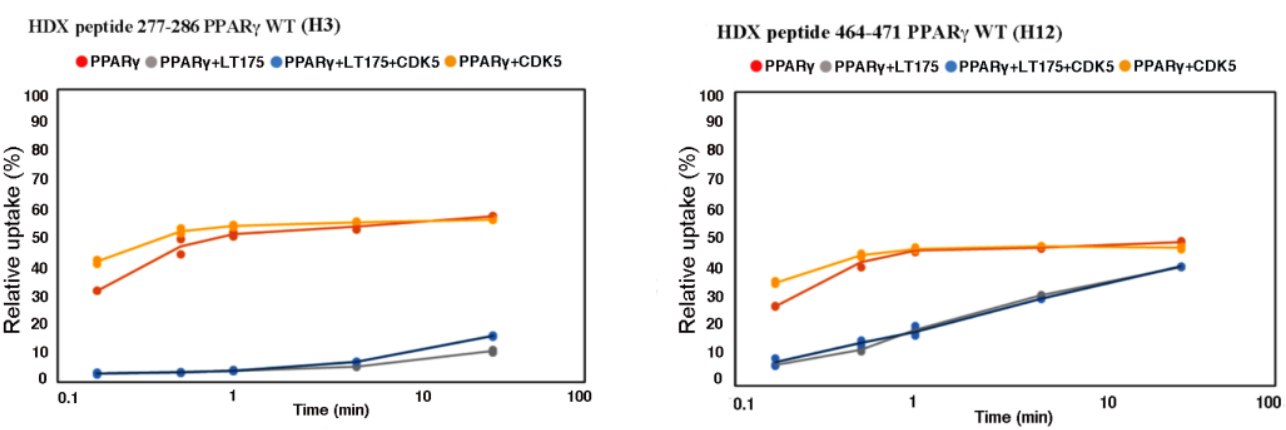

HDX peptide 277-286 PPAR I281A (H3)

-1281A -1281A+LT175 -1281A+LT175+CDK5 - I281A+CDK5

HDX peptide 464-471 PPAR $\gamma$ I281A (H12)

I281A I281A+LT175 -1281A+LT175+CDK5 I281A+CDK5
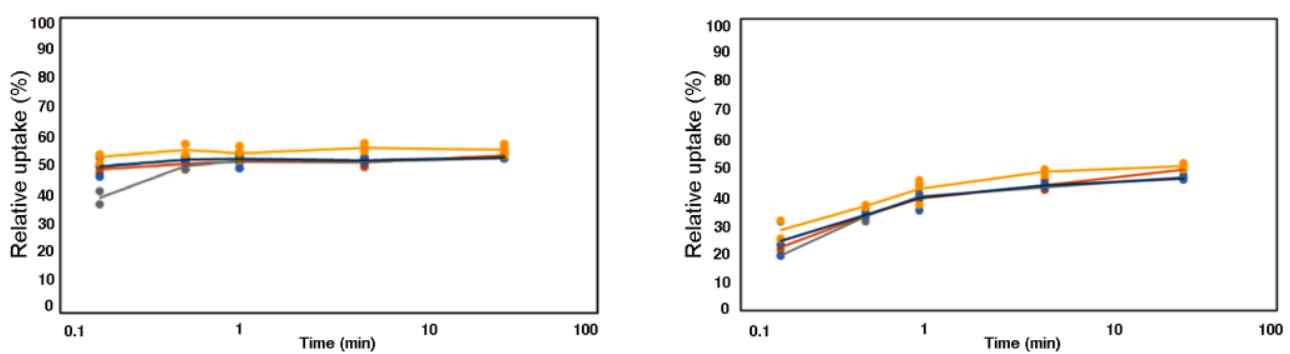

C

$\begin{array}{llllllllllllllll}T & G & \text { K } & \text { T } & \text { T } & \text { D } & \text { K } & \text { S } & \text { P } & \text { F } & \text { V } & \text { I } & \text { Y } & \text { D } & \text { M } & \text { N }\end{array}$
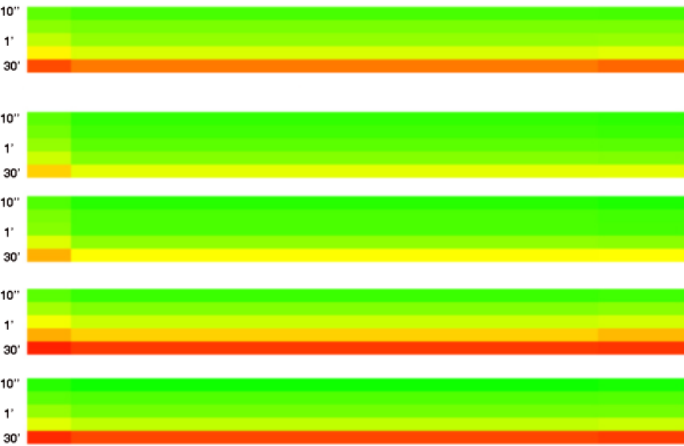

PPARY+LT175

PPARY +LT175+CDK5

PPARY+CDK5

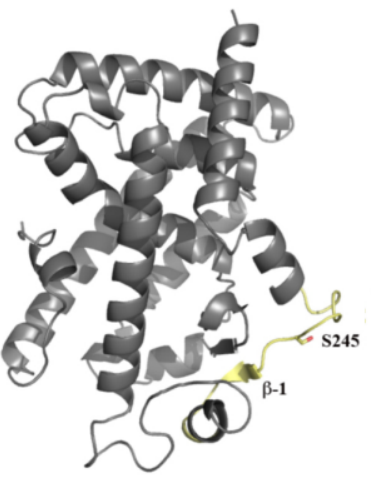

I281A

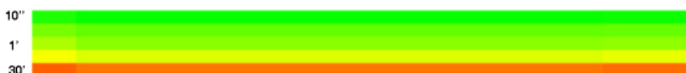

I281A+LT175

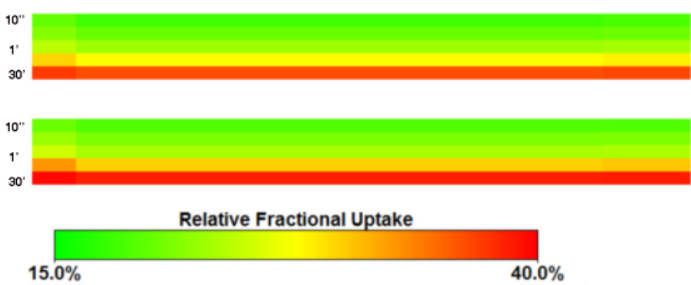

I281A+LT175+CDK5

$1281 \mathrm{~A}+\mathrm{CDK} 5$ 
Figure 9. HDX-MS analysis.

(a) Heat map showing a comparison between PPAR $\gamma$ in the presence or absence of LT175 where the relative uptake of PPAR $\gamma+\mathrm{LT} 175$ is subtracted from PPAR $\gamma$. The red areas represent an increased uptake, the green areas a decreased uptake, the yellow areas represent an unaffected uptake by the addition of LT175. Every bar is composed of 5 lines representing from top to bottom the time points 10 ', 30', 1', 5' and 30'.

(b) Deuterium relative uptake of peptides VAIRIFQGCQ (277-286) in PPAR $\gamma$ and VAIRAFQGCQ (277-286) in mutated PPAR $\gamma$ I281A and SLHPLLQE (464-471).

(c) Highlight on the segment containing S245 in all the tested conditions.

\section{LT175 doesn't bind the alternate site of PPAR $\gamma$}

As known, some PPAR $\gamma$ ligands are able to bind an alternate low-affinity site, expecially at high concentrations ${ }^{29-33}$. To exclude that the inhibition of phosphorylation by LT175 was caused by the binding of the ligand to the alternate site, close to the $\beta$-sheet, and not by the described allosteric mechanism, we set up the following experiment. The small ligand GW9662 $2^{30}$, known to covalently bind PPAR $\gamma$ to C285 preventing in this way the canonical binding of LT175 (Supporting Information Figure 13), was preventively equilibrated with PPAR $\gamma$. An SPR experiment was then performed, after immobilizing PPAR $\gamma /$ GW9662 on the chip, allowing LT175 to flow into the cell at the concentrations of $0.1,1,10$ and $100 \mu \mathrm{M}$. The sensograms showed that at the concentrations used in the phosphorylation experiments $(0.1,1$ and $10 \mu \mathrm{M})$ LT175 doesn't bind PPAR $\gamma$, and only at the high concentration of $100 \mu \mathrm{M}$ is able to bind the low affinity alternate site (Supporting Information Figure 14).

\section{Discussion}


This work extends several of our previous studies where we evidenced new structural features of PPAR $\gamma$ in the crystal complex with a new series of ligands that, interacting with different regions of the LBD, confer a differentiated biological response in cell and animal models ${ }^{34-39}$.

In particular, we provided a rational explanation of how some PPAR $\gamma$ ligands affect the CDK5-induced phosphorylation rate by increasing the stability of the $\beta$-sheet and decreasing the dynamic nature of the nuclear receptor depending on their binding mode.

To elucidate this mechanism, we explored in deep the dynamics of the CDK5 approach to PPAR $\gamma$ consensus region through the integration of a number of biophysical techniques including NMR and Hydrogen/deuterium exchange/mass spectrometry (HDX-MS), and observed how the ligand binding affects the inhibition rate of CDK5-mediated PPAR $\gamma$ phosphorylation.

In particular, we started from the hypothesis, suggested by the docking simulation, that the accommodation of PPAR $\gamma$ consensus region in the active site of CDK5 is possible only if a transitory unfolding of the PPAR $\gamma$-LBD $\beta 1$ strand occurs. The partial unfolding of $\beta 1$ is further supported by the observation that in the PPAR $\gamma$-homologous nuclear receptor $\mathrm{RAR}^{40}$ and in almost all nuclear receptors the corresponding $\beta$-strand is replaced by a loop (17 residues in the case of RAR), thus suggesting that this region is not essential for the global stability of the LBD.

As a consequence of this premise, the increase in the stability of the $\beta$-sheet should correspond to a decrease in S245 phosphorylation. 
A double mutant, designed to have a disulphide bridge that strongly stabilizes the $\beta$-sheet, confirmed that the phosphorylation of S245 is completely inhibited if the transient unfolding of $\beta 1$ strand by $\mathrm{Cdk} 5$ is prevented. As shown by Ribeiro Filho et al. ${ }^{19}$, the stabilization of the helix H2' and the loop H2-H2' at the interface between PPAR $\gamma$ and Cdk5/p25 can also affect the phosphorylation rate.

This is in accordance with the observation that ligands bound at the hydrophobic region between $\mathrm{H} 3$ and $\beta 1-\beta 4$ of PPAR $\gamma$, such as $\mathbf{7 j}$, the partial agonists $(R)-1^{14}$, MRL2 $4^{12,17}$ and nTZDpa ${ }^{41}$, are able to reduce the mobility of this region, through vdW interactions (Supporting Information Figure 15) or H-bonds with the $\beta 3-\beta 4$ strand (S342), inhibiting the phosphorylation of S245 by CDK5.

Then, the direct stabilization of the $\beta$-sheet by interactions with residues of the internal strands of the $\beta$-sheet ( $\beta 3-\beta 4)$, the helix H2' and the loop H2H2', makes S245 less available for the kinase and represents the main strategy to block PPAR $\gamma$ phosphorylation. Also full agonists, such as rosiglitazone that possesses a terminal tail in front of $\beta 4$, are able to stabilize the $\beta$-sheet through vdW interactions (Supporting Information Figure 16) inhibiting the phosphorylation, although to a lesser extent. In this regard, it is interesting to note that the small-size antagonist of $\operatorname{PPAR} \gamma, \operatorname{SB} 1404^{29}$ (PDB 5DV6), covalently bound to $\mathrm{C} 285$ in the canonical binding pocket, is not long enough as rosiglitazone to reach $\beta 4$ and doesn't inhibit the phosphorylation of S245. Similarly, 3a, whose long aliphatic chain, unlike $\mathbf{7 j}$, doesn't face the $\beta$-sheet, inhibits the phosphorylation to a lesser extent than $\mathbf{7 j}$ (Figure 4, upper level). In the same way between the two enantiomers 
SB1494 and SB1495 ${ }^{42}$ (PDB 6IJS and 6IJR, respectively), the first bound in the region between $\mathrm{H} 3, \mathrm{H} 2$, the $\beta$-sheet and the $\omega$-loop, the second in a different region far from the $\beta$-sheet, only the first is able to inhibit the phosphorylation of S245 (Supporting Information Figure 17). On the contrary, less clear was the case of $\mathrm{LT} 175^{14}$ that, although occupying a region far from the consensus motif, also decreases the phosphorylation rate. Specifically, here we demonstrate that a long distance allosteric mechanism to block PPAR $\gamma$ phosphorylation is possible. Indeed, LT175 by occupying a new branch of the LBD, named 'diphenyl pocket', displaces the side-chain of F282 that, by switching from trans to gauche* upon ligand binding, triggers a hydrophobic cross-talk (Figure 7). This pathway passes through several hydrophobic residues belonging to $\mathrm{H} 3, \mathrm{H} 6$, and the $\beta$-sheet and arrives up to S245, making it less available to phosphorylation by CDK5. This hydrophobic cross-talk can also extend up to H2' and H2-H2' loop, via residues I341 and M348, altering their stability and preventing a proper interaction with $\mathrm{Cdk} 5 / \mathrm{p} 25$, with a consequent decreased phosphorylation ${ }^{19}$ (Figure 7 and Supporting Information Figure 2). The crucial role of M348 for the stabilization of the $\beta$-sheet, the $\mathrm{H} 2-\mathrm{H} 2$ ' loop and $\mathrm{H} 2$ ' helix was also confirmed by the marked change of its chemical shift, upon ligand binding, observed in the previously described NMR experiment.

It is interesting to note that in this allosteric mechanism a central role is played by $\mathrm{H} 3$ that allows distant regions to communicate with each other, as shown by both site-specific mutagenesis and HDX experiments. Although blocking of phosphorylation can be mediated by ligand allostery directly from ligand to the kinase binding surface of PPAR, there are also 
other inhibition mechanisms such as one proposed by Li et al. ${ }^{43}$ where NCoR tethers the kinase into the transcriptional complex.

\section{CONCLUSIONS}

In conclusion, in this work two different ways to alter the dynamics of the $\beta$-sheet affecting S245 phosphorylation have been shown:

i) a mechanism of more direct stabilization of the $\beta$-sheet, $\mathrm{H} 2$ ' and $\mathrm{H} 2-\mathrm{H} 2$ ' loop, as realized by most ligands with reduced agonist activity, such as MRL24, but also by full agonists, such as rosigitazone, long enough to interact with the $\beta 4$ strand;

ii) a long distance allosteric mechanism, as the case of the partial agonist LT175, by which a similar stabilization of the $\beta$-sheet can be obtained, leading to an equally effective inhibition of Ser 245 phosphorylation. It is evident that in both cases the rate of inhibition is not correlated to the degree of ligand agonism but rather to its ability to stabilize the $\beta$-sheet through $\mathrm{H}$ bonds or vdW interactions. As shown by Choi et al. ${ }^{11}$, this inhibition leads to the recruitment of a different set of coactivators that provokes an increase of the expression of insulin sensitizing genes, such as adiponectin. This may explain why PPAR $\gamma$ partial agonists, such as MRL24, or non-agonists can exhibit similar or higher antidiabetic effects than those of full agonists, whereas the classical agonism is not required for strong antidiabetic action but is rather associated to the occurrence of undesired side effects. These general considerations may provide a new structural strategy for designing of anti-diabetic drugs acting on PPAR $\gamma$. 


\section{Experimental Section}

\section{General information}

The compounds used in this work have a purity $\geq 95 \%$ (Table 3 of Supporting information).

\section{Site-directed mutagenesis}

The PPAR $\gamma$ I281A mutant was cloned in pET-28 plasmid for Escherichia coli expression. The QuickChange Site-Directed Mutagenesis Kit (Stratagene) was used to introduce the point mutation into the bacterial expression vector. The forward primer for the mutation I281A was: 5'-

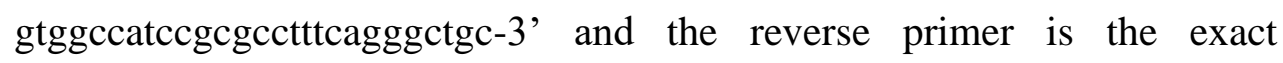
complement of the forwards primer. The PPAR $\gamma$ double mutant F247C/G346C was generated by two successive QuickChange Site-Directed Mutagenesis events to introduce the mutations. Sequences of the forward primers, with the altered residue underlined, were as follows: F247C 5'ggaaagacaacagacaaatcaccatgcgttatctatgacatgaatt-3'; G346C 5'-ctcatatccgagggccaatgcttcatgacaaggg-3'; the reverse primers used are the exact complements of the forwards primers. DNA sequencing was performed to confirm the presence of the desired mutations.

\section{Protein expression and purification}

The ligand-binding domain of human PPAR $\gamma$ (NP_001120802; aa 207474), I281A and the double mutant F247C/G346C were expressed as Nterminally His-tagged proteins using pET-28 vector and were purified as 
previously described $^{35}$. For NMR experiments, the expression plasmid was transformed into E. coli Rosetta (DE3) pLysS cells (Invitrogen) and grown at $37{ }^{\circ} \mathrm{C}$ in a M9-based medium containing $34 \mu \mathrm{g} / \mathrm{mL}$ kanamycin supplemented with ${ }^{15} \mathrm{NH}_{4} \mathrm{Cl}$ (Cambridge isotopes). When the cell density reached OD600nm $\sim 0.6$, protein expression was induced with $0.5 \mathrm{mM}$ isopropyl $\beta$-D-thiogalactoside (IPTG) and cells were grown for 18 hours at $20{ }^{\circ} \mathrm{C}$, harvested by centrifugation. Cells were lysed by sonication in buffer A containing $20 \mathrm{mM}$ Tris/Cl pH 8.0, $100 \mathrm{mM} \mathrm{NaCl,} 2 \mathrm{mM}$ TCEP, $1 \mathrm{mM}$ 4(2-Aminoethyl) benzenesulfonyl fluoride hydrochloride (AEBSF) and protease inhibitor cocktail (Nacalai Tesuque). After centrifugation the supernatant was applied to a Ni-NTA agarose column (QIAGEN), and the His-tagged protein was eluted with buffer A containing $250 \mathrm{mM}$ imidazole. The His-tag was removed by overnight digestion with TEV protease (Nacalai Tesuque) at $25^{\circ} \mathrm{C}$. The PPAR $\gamma$ LBD was further purified on a Resource Q column (GE Healthcare) using a $0-1.0 \mathrm{M} \mathrm{NaCl}$ gradient in buffer A followed by gel filtration on a Superdex S75 in $20 \mathrm{mM}$ Tris/Cl pH 8.0.

\section{NMR}

${ }^{1} \mathrm{H}-{ }^{15} \mathrm{~N}$-heteronuclear single quantum correlation (HSQC) spectrum were collected at $25^{\circ} \mathrm{C}$ on Bruker AV600 spectrometer equipped with a cryoprobe accessory. A NMR sample containing $0.3 \mathrm{mM}{ }^{15} \mathrm{~N}$ labeled PPAR $\gamma$ LBD, 0.03 to $0.3 \mathrm{mM}$ ligand-bound, labeled PPAR $\gamma$ LBD in a NMR buffer $\left(5 \% \mathrm{D}_{2} \mathrm{O}\right.$, $0.05 \%$ sodium azide, $20 \mathrm{mM}$ potassium phosphate, $\mathrm{pH}$ 7.4, $50 \mathrm{mM} \mathrm{KCl}, 8$ 
$\mathrm{mM} \beta$-mercaptoethanol- $d_{6}$ and $0.5 \mathrm{mM}$ EDTA- $\left.d_{16}\right)$ was prepared as described $^{44}$.

The chemical shift for Met348 was assigned using BMRB Entry $15518^{44}$ and $17975^{28}$

\section{In vitro kinase assay}

The assay was performed on the double mutant F247C/G346C, and both the WT-PPAR $\gamma$ and I281A-PPAR $\gamma$ LBDs, in the apo-form and in the complex with LT175 and other ligands (rosiglitazone, 7j, 3a, MRL24 and $(R)-\mathbf{1})$. For the kinase assay, stock solutions of ligands were prepared by diluting with $100 \%$ DMSO to a concentration of $500 \mu \mathrm{M}$. The stock solutions were further diluted with $50 \mathrm{mM}$ Tris $\mathrm{HCl} \mathrm{pH} 7.5$ up to the final concentration of $10 \mu \mathrm{M}$, and pre-equilibrated o.n. at $4^{\circ} \mathrm{C}$ with the protein. Kinase assay was carried out at $30^{\circ} \mathrm{C}$ for 2 hours in $50 \mu$ volume buffer containing 50mM Tris $\mathrm{HCl} \mathrm{pH} 7.5,1.2 \mu \mathrm{g}$ PPAR $\gamma, 10 \mu \mathrm{M}$ ligand, $2.5 \mathrm{mM}$

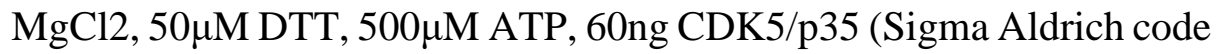
n. SRP5011).

\section{Pro-Q Diamond phosphoprotein gel stain}

Pro-Q Diamond Phosphoprotein Gel Stain provides a convenient method for selectively staining phosphoproteins in acrylamide gels, without the need for blotting or use of phosphoprotein specific antibodies. After performing electrophoresis and reading results with Pro-Q Diamond Phosphoprotein Gel Stain (ChemiDoc), a further step of gel staining with SYPRO Ruby protein gel stain was performed in order to ascertain the relative phosphorylation 
state of proteins for normalization. More detailed information on the protocol used in this experiment can be obtained at Thermofisher site.

\section{ELISA of PPAR $\gamma$ phosphorylation}

Polystyrene microwell plates (Nunc immuno-plate Maxisorp 96 well, Sigma code M9410) were coated with the reaction mixture.

After overnight incubation at $4{ }^{\circ} \mathrm{C}$, the coated wells were washed three times with WB (PBS + Tween $0.005 \%$ ) and left to block in PBS containing 1\% bovine serum for $90 \mathrm{~min}$ at $37^{\circ} \mathrm{C}$. The wells were then washed three times with WB and $100 \mathrm{ul}$ of anti-phospho-Ser/Thr-Pro antibody (Sigma Aldrich code n. A05368) diluted 1:500 in PBS was added to the wells and incubated for $60 \mathrm{~min}$ at $37^{\circ} \mathrm{C}$.

The wells were washed three times with WB and $100 \mu$ l of Anti-Mouse IgG (whole molecule)-Peroxidase antibody produced in goat (Sigma Aldrich code $n$. A4416) diluted 1:1000 were added to the wells. After 60 min of incubation at $37^{\circ} \mathrm{C}$, the wells were washed three times with WB and $200 \mu \mathrm{l}$ of $o$-Phenylenediamine dihydrochloride (Sigmafast OPD code n. P9187) dissolved in water were added to the wells. Optical density (OD) was measured at 450nm using Apply Scan Thermofisher Reader and the data were processed using Excel.

\section{Ellman's assay}

The Ellman's assay kit measures sulfhydryl groups with the thiol reagent 55dithiobis[2nitrobenzoic acid] (DTNB), which forms the 5-thionitrobenzoic acid and a mixed disulfide. Under conditions of oxidative stress, free 
sulfhydryls decrease and disulfides increase. Further information on the protocol used in the experiment can be obtained consulting the Thermo Scientific site.

\section{CD analyses}

The CD spectra of PPAR $\gamma$ wild type and the double mutant F247C/G346C, both in the reduced (+ TCEP) and oxidized form, were recorded at $20{ }^{\circ} \mathrm{C}$ using a Jasco J-810 spectropolarimeter equipped with a Peltier thermostatic cell holder. Far-UV measurements (190-260 nm) were carried out using a 0.1-cm path length cell in $20 \mathrm{mM}$ Tris $\mathrm{HCl}, 0.5 \mathrm{mM}$ EDTA $\mathrm{pH} 7.5$ at a protein concentration of $10 \mu \mathrm{M}$.

\section{GuHCl denaturation experiment}

GuHCl ( $\geqq 99 \%)$ was purchased from Nacalai Tesque (Kyoto Japan). In each $\mathrm{GuHCl}$ denaturation experiment, samples of $\operatorname{PPAR} \gamma$ were titrated with $\mathrm{GuHCl}$ from 0 to $6.0 \mathrm{M}$, at a protein concentration of $20 \mu \mathrm{M}$. Unfolding was intiated by dilution of a concentrated protein stock into the appropriate $\mathrm{GuHCl}$ buffer. All samples were incubated at $20{ }^{\circ} \mathrm{C}$ for $2 \mathrm{~h}$ before measurement using JASCO J-1500 CD spectrometer.

\section{Model of the complex CDK5/p25/ATP/substrate and protein-protein docking}

To generate a model for the complex CDK5/p25/ATP, the homologous protein CDK2 (PDB code 1QMZ) was chosen as template for comparative 
modeling ${ }^{45}$ of the target protein CDK5 because it is the only known crystallographic structure of CDKs family available in the PDB in the complex with a substrate. Moreover, CDK2 and CDK5 share almost $60 \%$ of sequence identity in different species ${ }^{22}$. After cleaning the PDB files, the sequence of the target protein was threaded onto the three-dimensional backbone of the template structure according to the sequence alignment of the two proteins. Areas in which the template and the target sequence diverged substantially were remodeled and refined by using the Rosetta loop-building CCD algorithm. Loop coordinates for missing density in the threaded model were generated from fragment libraries obtained from both Robetta (http://robetta.bakerlab.org/fragmentsubmit.jsp) and Fragment Picker applications. To energetically minimize the Rosetta model, it has been followed the Rosetta relax protocol. Once the model of the target protein CDK5 was built, to generate the model of CDK5/p25/ATP/substrate, the peptide described by Brown et al. ${ }^{21}$ was used as template for the substrate and the file saved as PDB. The superposition was performed by $\mathrm{PyMol}^{46}$ and the energy minimization by MOE software ${ }^{47}$.

Once a putative model for the complex CDK5/p25/ATP/substrate was created, it was manually superposed by PyMol to its docking partner PPAR $\gamma$ so that the segment containing the residues S245 and P246 was exactly superimposed to the consensus peptide ASP described by Brown et al. ${ }^{21}$. A new file containing both the complex between CDK5/p25/ATP and its docking partner PPAR $\gamma$ was thus created and Rosetta v3.4 software was used to predict the bound structure of PPAR $\gamma / \mathrm{CDK} 5 / \mathrm{p} 25 / \mathrm{ATP}$ complex starting from the model. To run the Rosetta protocol for protein- 
protein docking a distance constraint file between PPAR $\gamma-\mathrm{S} 245$ and CDK5-K128 needed to be created. The position found in the lowresolution search was optimized by rigid body Monte Carlo Minimization before running the high-resolution docking.

\section{Molecular Dynamics}

All simulations have been performed using Gromacs 2016.1. The structures were centered in cubic boxes with minimum distance of $1.5 \mathrm{~nm}$ between each atom of the protein and the box. The SPC water model was used to solvate the systems. Ionic strength was adjusted so as to make sure all simulation were electrically neutral. MD simulations were performed with periodic boundary conditions in the isothermal-isochoric ensemble (NVT), using an integration step of $2 \mathrm{fs}$ and keeping the temperature constant at $300 \mathrm{~K}$ by using the velocity rescaling algorithm ${ }^{48}$. Electrostatic interactions were treated using the particle mesh Ewald method $^{49}$ for the long-range contribution (reciprocal space) and with a cut-off radius of $1.0 \mathrm{~nm}$. The Amber99b force field ${ }^{50}$ was used. Before production runs, all systems were subject to a minimization cycle and thermalization procedure to bring gradually the temperature to $300 \mathrm{~K}$. All runs consisted of at least $190 \mathrm{~ns}$ MD simulations in a NVT ensemble. Essential Dynamics analysis was performed according to Amadei et al. ${ }^{51}$. Basically, atomic positional fluctuations covariance matrix was built on the equilibrated portion of all trajectories and diagonalized to get principal components representing large amplitude motions sampled during simulation time. Trajectories were projected onto selected 
eigenvectors to show main dominant protein motions. Figures were generated using the Visual Molecular Dynamics software (http://www.ks.uiuc.edu/Research/vmd/).

\section{Hydrogen/Deuterium exchange coupled with Mass Spectrometry}

For HDX analysis, 60 pmol PPAR $\gamma$ were diluted into D2O according to the scheme in SI appendix. Samples were incubated for 10 seconds, 30 seconds, $1 \mathrm{~min}, 5 \mathrm{~min}$ and $30 \mathrm{~min}$ and performed at least in duplicate. The reaction was quenched by $1: 1$ dilution into a $0^{\circ} \mathrm{C}$ solution of $4 \mathrm{M}$ Urea, $200 \mathrm{mM}$ TCEP, with $\mathrm{pH}$ adjusted to give a final $\mathrm{pH}$ of 2.5. The quenched reaction was immediately injected into a Waters HDX/nanoAcquity system for digestion on an online pepsin column $\left(20^{\circ} \mathrm{C}\right.$, flow-rate $\left.125 \mu \mathrm{L} / \mathrm{min}\right)$ followed by separation on a $10 \mathrm{~min}$ RP-HPLC gradient $\left(0.5^{\circ} \mathrm{C}\right.$, flow-rate $\left.40 \mu \mathrm{L} / \mathrm{min}\right)$. The eluent was directed into a Xevo G2 instrument (Waters) with electrospray ionization and lock-mass correction using leu-enkephalin peptide. The online pepsin column used was an Applied Biosystems immobilized pepsin cartridge $(2.1 \mathrm{~mm}$ x $30 \mathrm{~mm})$. RP-HPLC column used was a Waters C18-BEH, $1.0 \times 100 \mathrm{~mm}$, with $1.7 \mu \mathrm{m}$ particles. Electrospray ionization was achieved with a capillary voltage of $3 \mathrm{kV}$ in conjunction with a cone voltage of $75 \mathrm{~V}$ and source temperature of $100^{\circ} \mathrm{C}$. Mass calibration was performed with sodium cesium iodide clusters up to $\mathrm{m} / \mathrm{z} 2000$, giving a mass accuracy $<5 \mathrm{ppm}$. Two injections of $2 \mathrm{M}$ Urea with a $\mathrm{pH}$ adjusted to 2.5 were performed between each sample injection to prevent sample carry over. Each sample was analysed at least in duplicate for each time point. Non-deuterated PPAR $\gamma$ samples were analysed in the same way for peptide 
sequencing, using MSe data acquisition and data processing with ProteinLynx Global Server 2.5 software. Uptake of deuterium for each peptide was calculated compared with the non-deuterated control samples using Waters DynamX 3.0.0 software.

\section{Crystallization, data collection and structure determination}

PPAR $\gamma$ LBD was expressed as N-terminal His-tagged proteins using a pET28 vector and purified as previously described ${ }^{35}$.

Crystals of apo-PPAR $\gamma$ were obtained by vapor diffusion at $18^{\circ} \mathrm{C}$ using a sitting drop made by mixing $2 \mu \mathrm{L}$ of protein solution with $2 \mu \mathrm{L}$ of reservoir solution (0.8 M Na Citrate, $0.15 \mathrm{M}$ Tris, $\mathrm{pH} 8.0$ ). The crystals were soaked for three days in a storage solution (1.2 M Na Citrate, 0.15 M Tris, pH 8.0) containing the ligand 3a $(0.5 \mathrm{mM})$. The ligand dissolved in DMSO $(50 \mathrm{mM})$ was diluted in the storage solution so that the final concentration of DMSO was $1 \%$. The storage solution with glycerol $20 \%(\mathrm{v} / \mathrm{v})$ was used as cryoprotectant. Crystals $(0.10 \times 0.10 \mathrm{~mm})$ of PPAR $\gamma / \mathbf{3 a}$ belong to the space group $C 2$ with cell parameters shown in Table 4 of Supporting Information. $\mathrm{X}$-ray data of the complex PPAR $\gamma / \mathbf{3 a}$ were collected at $100 \mathrm{~K}$ under a nitrogen stream using synchrotron radiation (beamline ID30B at ESRF, Grenoble, France). The diffracted intensities were processed using the programs Mosflm ${ }^{52}$ and SCALA ${ }^{52}$. Structure solution was performed with $\mathrm{AMoRe} \mathrm{e}^{53}$, using the coordinates of PPAR $\gamma / 7 \mathbf{j}^{15}$ (PDB code 6QJ5) as the starting model. The coordinates were then refined with $\mathrm{CNS}^{54}$ and PHENIX $^{55}$ including data between 57.84 and $1.95 \AA$. The statistics of 
crystallographic data and refinement and the omit map around $\mathbf{3 a}$ are summarized in Table 4 and Figure 18 of Supporting Information.

\section{ASSOCIATED CONTENT}

\section{Supporting Information}

The Supporting Information is available free of charge at ...

Experimental protocol for the HDX experiments and HDX figures; X-ray crystal structures of SB1494, SB1495, rosiglitazone, GW9662, LT175, 7j, 3a, in PPAR $\gamma$ LBD and double mutant F247C/G346C structure; omit density map around 3a; SPR sensograms of LT175 and I281A; NMR titration experiments; ELISA phosphorylation assay.

Molecular formula string for $\mathbf{3 a}(\mathrm{CSV})$

\section{Accession Codes}

Coordinates and structure factors of the PPAR $\gamma$ complex with the compound 3a have been deposited in the Protein Data Bank under the accession code 6T9C. Authors will release the atomic coordinates and experimental data upon article publication.

\section{AUTHOR INFORMATION}

\section{Corresponding Authors}

Giorgio Pochetti - CNR, Monterotondo Stazione, Roma, Italy; orcid.org/0000-0002-3980-3180

Email: giorgio.pochetti@ic.cnr.it

Toshimasa Itoh - Showa Pharmaceutical University, Machida, Tokyo, Japan;

orcid.org/0000-0002-9925-5091

Email: titoh@ac.shoyaku.ac.jp 
Roberta Montanari - CNR, Monterotondo Stazione, Roma, Italy;

orcid.org/0000-0002-7533-5425

Email: roberta.montanari@ic.cnr.it

Other Authors

Davide Capelli - CNR, Monterotondo Stazione, Rome, Italy;

Keiko Yamamoto - Showa Pharmaceutical University, Machida, Tokyo, Japan;

Hirono Awaishima - Showa Pharmaceutical University, Machida, Tokyo, Japan;

Kimina Nishikata - Showa Pharmaceutical University, Machida, Tokyo, Japan;

Arjan Barendregt - Bijovet Center for Biomolecular Research and Utrecht Institute for Pharmaceutical Sciences, Utrecht, Netherlands

Albert J.R. Heck - Bijovet Center for Biomolecular Research and Utrecht Institute for Pharmaceutical Sciences, Utrecht, Netherlands

Fulvio Loiodice - University of Bari, Bari, Italy;

Fabio Altieri - Sapienza University of Rome, Rome, Italy;

Alessandro Paiardini - Sapienza University of Rome, Rome, Italy;

Alessandro Grottesi - CINECA of Rome, Rome, Italy;

Luciano Pirone - CNR, Napoli, Italy;

Emilia Pedone - CNR, Napoli, Italy;

Frank Peiretti - Aix Marseill University, Marseille, France

Jean Michel Brunel - Aix Marseill University, Marseille, France

\section{Author contributions}

T. I., R. M. and G. P. designed the research; T. I., E. P. and L. P. performed site-directed mutagenesis; D. C. expressed and purified WT, I281A and F247C/G346C PPAR $\gamma$; R. M. and A. P. performed the docking simulation;

T. I., H. A. and K. N. performed NMR experiments; K. Y. supervised NMR experiments; A. P. and A. G. performed MD simulations and interpreted the data; R. M. and G. P. designed the HDX experiment; A. B. executed and analysed the HDX experiment; A. J. R. H. supervised the HDX experiment; R. M. and G. P. performed the in vitro kinase assay and the ELISA and Phosphoprotein Gel Stain; F. A. helped in design of ELISA assay and data analysis; F. L. synthesized and provided the ligands; L. P. and E. P. performed the CD experiment on F247C/G346C and WT 
PPAR $\gamma$; T. I. performed the CD experiment with $\mathrm{GdmCl}$ on I281A and WT PPAR $\gamma$; J. M. B. and F. P. synthesized 3a; G. P., D. C. and R. M. performed the X-ray analysis of the complex PPAR $\gamma / \mathbf{3 a} ;$ R. M. and G. P. drafted the manuscript; all authors reviewed the final manuscript.

\title{
Declaration of interests
}

The authors declare no competing interests.

\section{Acknowledgements}

This work was supported by Instruct Integrating Biology (PID: 1579) for HDX experiments. AP and RM received support from Associazione Italiana Ricerca sul Cancro (AIRC, https://www.airc.it/) MFAG 20447.

\begin{abstract}
Abbreviations
PPAR, peroxisome proliferator-activated receptor; CDK5, cyclin dependent kinase 5; CDK2, cyclin dependent kinase 2; HDX, hydrogen deuterium exchange; MD, molecular dynamics; T2D, type 2 diabetes; ERK, extracellular signal-regulated kinase; LBD, ligand binding domain; RMSF, root mean square fluctuation; PDB, protein data bank; RAR, retinoic acid receptor; TCEP, tris(2-carboxyethyl)phosphine; ELISA, enzyme-linked immunosorbent assay; HSQC, heteronuclear single quantum coherence; SPR, surface plasmon resonance; ED, essential dynamics; WB, washing buffer; DTNB, 5-5dithiobis[2-nitrobenzoic acid];
\end{abstract}




\section{References}

(1) Berger, J.; Moller, D. E. The Mechanisms of Action of PPARs. Annu. Rev. Med. 2002, 53, 409-435.

(2) Berger, J. P.; Akiyama, T. E.; Meinke, P. T. PPARs: Therapeutic Targets for Metabolic Disease. Trends Pharmacol. Sci. 2005, 26, 244-251. (3) Kliewer, S. A.; Sundseth, S. S.; Jones, S. A.; Brown, P. J.; Wisely, G. B.; Koble, C. S.; Devchand, P.; Wahli, W.; Willson, T. M.; Lenhard, J. M.; Lehmann, J. M. Fatty Acids and Eicosanoids Regulate Gene Expression through Direct Interactions with Peroxisome Proliferator-Activated Receptors Alpha and Gamma. Proc. Natl. Acad. Sci. U S A 1997, 94, 43184323.

(4) Issemann, I.; Green, S. Activation of a Member of the Steroid Hormone Receptor Superfamily by Peroxisome Proliferators. Nature 1990, $347,645-650$.

(5) Evans, R. M.; Barish, G. D.; Wang, Y. X. PPARs and the Complex Journey to Obesity. Na.t Med. 2004, 10, 355-361.

(6) Braissant, O.; Foufelle, F.; Scotto, C.; Dauca, M.; Wahli, W. Differential Expression of Peroxisome Proliferator-Activated Receptors (PPARs): Tissue Distribution of PPAR-Alpha, -Beta, and -Gamma in the Adult Rat. Endocrinology 1996, 137, 354-366.

(7) Forman, B. M.; Chen, J.; Evans, R. M. The Peroxisome ProliferatorActivated Receptors: Ligands and Activators. Ann. N Y Acad. Sci. 1996, 804, 266-275. 
(8) Willson, T. M.; Brown, P. J.; Sternbach, D. D.; Henke, B. R. The PPARs: from Orphan Receptors to Drug Discovery. J. Med. Chem. 2000, $43,527-550$.

(9) Bodmer, M.; Meier, C.; Kraenzlin, M. E.; Meier, C. R. Risk of Fractures with Glitazones: a Critical Review of the Evidence to Date. Drug Saf. 2009, 32, 539-547.

(10) Nesto, R. W.; Bell, D.; Bonow, R. O.; Fonseca, V.; Grundy, S. M.; Horton, E. S.; Le Winter, M.; Porte, D.; Semenkovich, C. F.; Smith, S.; Young, L. H.; Kahn, R. Thiazolidinedione Use, Fluid Retention, and Congestive Heart Failure: a Consensus Statement from the American Heart Association and American Diabetes Association. Diabetes Care 2004, 27, 256-263.

(11) Choi, J. H.; Banks, A. S.; Estall, J. L.; Kajimura, S.; Boström, P.;Laznik, D.; Ruas, J. L.; Chalmers, M. J.; Kamenecka, T. M.; Blüher, M.; Griffin, P. R.; Spiegelman, B. M. Anti-Diabetic Drugs Inhibit ObesityLinked Phosphorylation of PPARgamma by Cdk5. Nature 2010, 466, 451456.

(12) Choi, J. H.; Banks, A. S.; Kamenecka, T. M.; Busby, S. A.; Chalmers, M. J.; Kumar, N.; Kuruvilla, D. S.; Shin, Y.; He, Y.; Bruning, J. B.; Marciano, D. P.; Cameron, M. D.; Laznik, D.; Jurczak, M. J.; Schürer, S. C.; Vidović, D.; Shulman, G. I.; Spiegelman, B. M.; Griffin, P. R. Antidiabetic Actions of a Non-Agonist PPARgamma Ligand Blocking Cdk5-Mediated Phosphorylation. Nature 2011, 477, 477-481. Banks, A. S.; McAllister, S. E.; Camporez, J. P.; Zushin, P. J.;

Jurczak, M. J.; Laznik-Bogoslavski, D.; Shulman, G. I.; Gygi, S. P.; 
Spiegelman, B. M. An Erk/Cdk5 Axis Controls the Diabetogenic Actions of PPAR $\gamma$. Nature 2015, 517, 391-395.

(14) Montanari, R.; Saccoccia, F.; Scotti, E.; Crestani, M.; Godio, C.;

Gilardi, F.; Loiodice, F.; Fracchiolla, G.; Laghezza, A.; Tortorella, P.;

Lavecchia, A.; Novellino, E.; Mazza, F.; Aschi, M.; Pochetti, G. Crystal Structure of the Peroxisome Proliferator-Activated Receptor Gamma (PPARgamma) Ligand Binding Domain Complexed with a Novel Partial Agonist: a New Region of the Hydrophobic Pocket Could Be Exploited for Drug Design. J. Med. Chem. 2008, 51, 7768-7776.

(15) Peiretti, F.; Montanari, R.; Capelli, D.; Bonardo, B.; Colson, C.;

Amri, E.-Z.; Grimaldi, M.; Balaguer, P.; Pochetti, G.; Brunel, J. M. A

Novel N-Substituted Valine Derivative with Unique PPAR $\gamma$ Binding

Properties and Biological Activities. J. Med. Chem. 2020 (submitted).

(16) Gilardi, F.; Giudici, M.; Mitro, M.; Maschi, O.; Guerrini, U.; Rando, G.; Maggi, A.; Cermenati, G.; Laghezza, A.; Loiodice, F.; Pochetti, G.; Lavecchia, A.; Caruso, D.; De Fabiani, E.; Bamberg, K.; Crestani, M. LT175 is a Novel PPAR $\alpha / \gamma$ Ligand with Potent Insulin-Sensitizing Effects and Reduced Adipogenic Properties. J. Biol Chem. 2014, 289, 6908-6920.

(17) Hughes, T. S.; Giri, P. K.; de Vera, I. M. S.; Marciano, D. P.; Kuruvilla, D. S.; Shin, Y.; Blayo, A.-L.; Kamenecka, T. M.; Burris, T. P.; Griffin, P. R.; Kojetin, D. J. An Alternate Binding Site for PPARgamma ligands. Nat. Commun. 2014, 5, 3571.

(18) Mottin, M.; Souza, P. C.; Skaf, M. S. Molecular Recognition of PPARgamma by Kinase Cdk5/p25: Insights from a Combination of Protein- 
Protein Docking and Adaptive Biasing Force Simulations. J. Phys. Chem. B 2015, 119, 8330-8339.

(19) Ribeiro Filho, H. V.; Guerra, J. V.; Cagliari, R.; Batista, F. A. H.; Le Maire, A.; Oliveira, P. S. L.; Figueira, A. C. M. Exploring the Mechanism of PPAR $\gamma$ Phosphorylation Mediated by CDK5. J. Struct. Biol. 2019, 207, 317-326.

(20) Duarte, M. I.; Pena, D. A.; Nunes Ferraz, F. A.; Berti, D. A.; Paschoal Sobreira, T. J.; Costa-Junior, H. M.; Abdel Baqui, M. M.; Disatnik, M.-H.; Xavier-Neto, J.; Lopes de Oliveira, P. S.; Schechtman, D. Protein Folding Creates Structure-Based, Noncontiguous Consensus Phosphorylation Motifs Recognized by Kinases. Sci. Signal 2014, 7, ra105.

(21) Brown, N. R.; Noble, M. E.; Endicott, J. A.; Johnson, L. N. The Structural Basis for Specificity of Substrate and Recruitment Peptides for Cyclin-Dependent Kinases. Nat Cell Biol 1999, 1, 438-443.

(22) Tarricone, C.; Dhavan, R.; Peng, J.; Areces, L. B.; Tsai, L. H.; Musacchio, A. Structure and Regulation of the CDK5-p25(nck5a) Complex. Mo.l Cell 2001, 8, 657-669.

(23) Kaufmann, K. W.; Lemmon, G. H.; Deluca, S. L.; Sheehan, J. H.; Meiler, J. Practically Useful: what the Rosetta Protein Modeling Suite Can do for You. Biochemistry 2010, 49, 2987-2998.

(24) Ruoppolo, M.; Lundstrom-Ljung, J.; Talamo, F.; Pucci, P.; Marino, G. Effect of Glutaredoxin and Protein Disulfide Isomerase on the Glutathione-Dependent Folding of Ribonuclease A. Biochemistry 1997, 36, 12259-12267. 
(25) Nolte, R. T.; Wisely, G. B.; Westin, S.; Cobb, J. E.; Lambert, M. H.;

Kurokawa, R.; Rosenfeld, M. G.; Willson, T. M.; Glass, C. K.; Milburn, M.

V. Ligand Binding and Co-Activator Assembly of the Peroxisome Proliferator-Activated Receptor-Gamma. Nature 1998, 395, 137-143.

(26) Chrisman, I. M.; Mou, T. C.; Sprang, S. R.; Hughes, T.

S. PPARgamma LBD Complexed with GW1929 (PDB 6D8X) (to be published)

(27) Hughes, T. S.; Chalmers, M. J.; Novick, S.; Kuruvilla, D. S.; Chang, M. R.; Kamenecka, T. M.; Rance, M.; Johnson, B. A.; Burris, T. P.; Griffin, P. R.; Kojetin, D. J. Ligand and Receptor Dynamics Contribute to the Mechanism of Graded PPARgamma Agonism. Structure 2012, 20, 139-150. (28) Hamuro, Y.; Coales, S. J.; Morrow, J. A.; Molnar, K. S.; Tuske, S. J.; Southern, M. R.; Griffin, P. R. Hydrogen/Deuterium-Exchange (H/D-Ex) of PPARgamma LBD in the Presence of Various Modulators. Protein Sci. 2006, 15, 1883-1892.

(29) Bae, H.; Jang, J. Y.; Choi, S.-S.; Lee, J.-J.; Kim, H.; Jo, A.; Lee, K.J.; Choi, J.-H.; Suh, S. W.; Park, S. B. Mechanistic Elucidation Guided by Covalent Inhibitors for the Development of Anti-Diabetic PPAR $\gamma$ Ligands. Chem. Sci. 2016, 7, 5523-5529.

(30) Brust, R.; Lin, H.; Fuhrmann, J.; Asteian, A.; Kamenecka, T. M.; Kojetin, D. J. Modification of the Orthosteric PPARgamma Covalent Antagonist Scaffold Yealds an Improved Dual-Site Allosteric Inhibitor. ACS Chem. Biol. 2017, 12, 969-978.

(31) Hughes, T. S.; Shang, J.; Brust, R.; de Vera, I. M. S.; Fuhrmann, J.; Ruiz, C., Cameron, M. D.; Kamenecka, T. M.; Kojetin, D. J. Probing the 
Complex Binding Modes of the PPARgamma Partial Agonist 2-chloro-N(3-chloro-4-((5-chlorobenzo[d]thiazol-2-yl)thio)phenyl)-4-

(trifluoromethyl)benzenesulfonamide (T2384) to Orthosteric and Allosteric Sites with NMR Spectroscopy. J. Med. Chem. 2016, 59,10335-10341.

(32) Jang, J. Y.; Koh, M.; Bae, H.; An, D. R.; Im, H. N.; Kim, H. S.; Yoon, J. Y.; Han, B. W.; Park, S. B.; Suh, S. W. Structural Basis for Differential Activities of Enantiomeric PPARgamma Agonists: Binding of S35 to the Alternate Site. Biochim. Biophy.s Acta Proteins Proteom. 2017, 1865, 674681.

(33) Laghezza, A.; Piemontese, L.; Cerchia, C.; Montanari, R.; Capelli, D.; Giudici, M.; Crestani, M.; Tortorella, P.; Peiretti, F.; Pochetti, G.; Lavecchia, A.; Loiodice, F. Identification of the First PPARalpha/gamma Dual Agonist Able to Bind to Canonical and Alternative Sites of PPARgamma and to Inhibit its Cdk5-mediated Phosphorylation. J. Med. Chem. 2018, 61, 8282-8298.

(34) Pochetti, G.; Godio, C.; Mitro, N.; Caruso, D.; Galmozzi, A.; Scurati, F.; Loiodice, F.; Fracchiolla, G.; Tortorella, P.; Laghezza, A.; Lavecchia, A; Novellino, E.; Mazza, F.; Crestani, M. Insights into the Mechanism of Partial Agonism: Crystal Structures of the Peroxisome Proliferator-Activated Receptor Gamma Ligand-Binding Domain in the Complex with two Enantiomeric Ligands. J. Bio.l Chem. 2007, 282, 1731417324.

(35) Fracchiolla, G.; Laghezza, A.; Piemontese, L.; Tortorella, P.; Mazza, F.; Montanari, R.; Pochetti, G.; Lavecchia, A.; Novellino, E.; Pierno, S.; Conte Camerino D.; Loiodice, F. New 2-aryloxy-3-phenyl-propanoic acids 
Agonists with Improved Potency and Reduced Adverse Effects on Skeletal Muscle Function. J. Med. Chem. 2009, 52, 6382-6393.

(36) Pochetti, G.; Mitro, N.; Lavecchia, A.; Gilardi, F.; Besker, N.; Scotti, E.; Aschi, M.; Re, N.; Fracchiolla, G.; Laghezza, A.; Tortorella, P.; Montanari, R.; Novellino, E.; Mazza, F.; Crestani, M.; Loiodice, F. Structural Insight into Peroxisome Proliferator-Activated Receptor Gamma Binding of two Ureidofibrate-Like Enantiomers by Molecular Dynamics, Cofactor Interaction Analysis, and Site-Directed Mutagenesis. J. Med. Chem. 2010, 53, 4354-4366.

(37) Porcelli, L.; Gilardi, F.; Laghezza, A.; Piemontese, L.; Nitro, N.; Azzariti, A.; Altieri, F.; Cervoni, L.; Fracchiolla, G.; Giudici, M.; Guerrini, U.; Lavecchia, A.; Montanari, R.; Di Giovanni, C.; Paradiso, A.; Pochetti, G.; Simone, G. M.; Tortorella, P.; Crestani, M.; Loiodice, F. Synthesis, Characterization and Biological Evaluation of Ureidofibrate-Like Derivatives Endowed with Peroxisome Proliferator-Activated Receptor Activity. J. Med. Chem. 2012, 55, 37-54.

(38) Fracchiolla, G.; Laghezza, A.; Piemontese, L.; Parente, M.; Lavecchia, A.; Pochetti, G.; Montanari, R.; Di Giovanni, C.; Carbonara, G.; Tortorella, P.; Novellino, E.; Loiodice, F. Synthesis, Biological Evaluation and Molecular Investigation of Fluorinated Peroxisome ProliferatorActivated Receptors alpha/gamma Dual Agonists. Bioorg. Med. Chem. 2012, 20, 2141-2151.

(39) Laghezza, A.; Pochetti, G.; Lavecchia, A.; Fracchiolla, G.; Faliti, S.; Piemontese, L.; Di Giovanni, C.; Iacobazzi, V.; Infantino, V.; Montanari, R.; 
Capelli, D.; Tortorella, P.; Loiodice, F. New 2-(aryloxy)-3-phenylpropanoic Acids as Peroxisome Proliferator-Activated Receptor alpha/gamma Dual Agonists Able to Upregulate Mitochondrial Carnitine Shuttle System Gene Expression. J. Med. Chem. 2013, 56, 60-72.

(40) Bourguet, W.; Vivat, V.; Wurtz, J. M.; Chambon, P.; Gronemeyer, H.; Moras, D. Crystal Structure of a Heterodimeric Complex of RAR and RXR Ligand-Binding Domains. Mol Cell 2000, 5, 289-298.

(41) Bruning, J. B.; Chalmers, M. J.; Prasad, S.; Busby, S. A.; Kamenecka, T. M.; He, Y.; Nettles, K. W.; Griffin, P. R. Crystal Structure of PPARgamma Bound to Partial Agonist nTZDpa. Structure 2007, 15, 12581271.

(42) Jang, J.Y.; Kim, H.; Kim, H.-J.; Suh, S. W.; Park, S. B.; Han, B. W. Structural Basis for the Inhibitory Effects of a Novel Reversible Covalent Ligand on PPAR $\gamma$ Phosphorylation. Sci. Rep 2019, 9, 11168.

$$
\text { Li, P.; Fan, W.; Xu, J.; Lu, M.; Yamamoto, H.; Auwerx, J.; Sears, }
$$

D. D.; Talukdar, S.; Oh, D.; Chen, A.; Bandyopadhyay, G.; Scadeng, M.;

Ofrecio, J. M.; Nalbandian, S.; Olefsky, J. M. Adipocyte NCoR Knockout Decreases PPAR $\gamma$ Phosphorylation and Enhances PPAR $\gamma$ Activity and Insulin Sensitivity. Cell 2011, 147, 815-826.

(44) Lu, J.; Chen, M.; Stanley, S. E.; Li, E. Effect of Heterodimer Partner RXRalpha on PPARgamma Activation Function-2 Helix in Solution. Biochem. Biophy.s Res. Commun. 2008, 365, 42-46.

(45) Combs, S.A.; Deluca, S. L.; Deluca, S. H.; Lemmon, G. H.; Nannemann, D. P.; Nguyen, E. D.; Willis, J. R.; Sheehan, J. H.; Meller, J. 
Small-Molecule Ligand Docking into Comparative Models with Rosetta. Nat. Protoc. 2013, 8, 1277-1298.

(46) The PyMOL Molecular Graphics System, Version 2.0 Schroedinger, LLC.

(47) Molecular Operating Environment (MOE), 2013.08; Chemical Computing Group ULC, 1010 Sherbooke St. West, Suite \#910, Montreal, QC, Canada, H3A 2R7, 2018.

(48) Bussi, G.; Donadio, D.; Parrinello, M. Canonical Sampling through Velocity Rescaling. J. Chem. Phys. 2007, 126, 014101.

(49) Di Pierro, M.; Elber, R.; Leimkuhler, B. A Stochastic Algorithm for the Isobaric-Isothermal Ensemble with Ewald Summations for all Long Range Forces. Journal of Chemical Theory and Computation 2015, 11.

(50) Chen, A. A.; Pappu, R. V. Parameters of Monovalent Ions in the AMBER-99 Forcefield: Assessment of Inaccuracies and Proposed Improvements. J. Phys. Chem. B 2007, 111, 11884-11887.

(51) Amadei, A.; Linnsen, A. B. M.; Berendsen, H. J. C. Essential Dynamics of Proteins. Proteins 1993, 17, 412-425.

(52) Leslie, A. G. W. Joint CCP4and ESFEACMB Newslwtter on Protein. Protein Crystallogr. 1992, 26, 27-33.

(53) Navaza, J. Acta Crystallogr. Sect. A 1994, 50, 157-163.

(54) Brunger, A. T.; Adams, P. D.; Clore, G. M.; DeLano, W. L.; Gros, P.; Grosse-Kunstleve, R. W.; Jiang, J. S.; Kuszewski, J.; Nilges, M.; Pannu, N. S.; Read, R. J.; Rice, L. M.; Simonson, T.; Warren, G. L. Acta Crystallogr. Section D, 1998, 54 (Part 5), 905-921. 
(55) Adams, P. D.; Afonine, P. V.; Bunkoczi, G.; Chen, V. B.; Davis, I. W.; Echols, N.; Headd, J. J.; Hung, L.-W.; Kapral, G. J.; Grosse-Kunstleve, R. W.; McCoy, A. J.; Moriarty, N. W.; Oeffner, R.; Read, R. J.; Richardson, D. C.; Richardson, J. S.; Terwilliger, T. C.; Zwart, P. H. Acta Crystallogr. Sect. D, 2010, 66, 213-221. 